Geometry $\&$ Topology

Volume 8 (2004) 779-830

Published: 20 May 2004

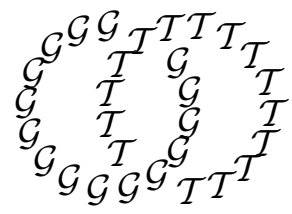

\title{
ASD moduli spaces over four-manifolds with tree-like ends
}

\author{
Tsuyoshi Kato \\ Department of Mathematics, Kyoto University \\ Kyoto 606-8502, Japan \\ Email: tkato@math.kyoto-u.ac.jp
}

\begin{abstract}
In this paper we construct Riemannian metrics and weight functions over Casson handles. We show that the corresponding Atiyah-Hitchin-Singer complexes are Fredholm for some class of Casson handles of bounded type. Using these, the Yang-Mills moduli spaces are constructed as finite dimensional smooth manifolds over Casson handles in the class.
\end{abstract}

\section{AMS Classification numbers Primary: 57M30, 57R57}

Secondary: $14 \mathrm{~J} 80$

Keywords: Yang-Mills theory, Casson handles

Proposed: Simon Donaldson

Seconded: Ronald Stern, Robion Kirby
Received: 16 October 2001

Revised: 29 March 2004 


\section{Introduction}

\section{A Review of previous works on exotic smooth structures on open four-manifolds}

Four-manifold theory has been deeply developed from two approaches. One is based on geometry and functional analysis. In particular gauge theory, YangMills theory or Seiberg-Witten theory, construct moduli spaces as the sets of solutions of nonlinear PDEs on four-manifolds. They have been discovered to contain extremely rich information on smooth structure. In particular the construction of Donaldson's invariant uses Yang-Mills moduli spaces. The invariant distinguishes many mutually-homeomorphic but non-diffeomorphic pairs of smooth four-manifolds. The other is based on topology, in particular CassonFreedman's theory. In high dimensional differential topology, Whitney's trick to remove self-intersections of immersed discs has played one of the most important rôles, but does not work in four dimensions. Casson's idea was, instead of removing them, to increase self-intersections by attaching immersed two handles constructively so that all self-intersections were able to be pushed away to infinity. Freedman verified that these infinite towers made from immersed two-handles were all homeomorphic to the standard open two-handle, and they are called Casson handles. This allowed the complete classification of oriented simply-connected topological four-manifolds by their intersection forms and Kirby-Siebenmann classes [15].

Combination of Casson-Freedman's theory with gauge theory provided very deep results on smooth structures on four-manifolds. One of the most important results on exotic $\mathbb{R}^{4}$ s was due to Taubes, who discovered uncountably many exotic smooth structures on $\mathbb{R}^{4}[27$. In the essential step, he constructed Yang-Mills gauge theory on periodic open four-manifolds. Let $X$ be an oriented topological four-manifold. $X \backslash p t$ can admit at least one smooth structure [16. The idea was to verify that $X \backslash p t$ was not able to admit any end-periodic smooth structure. In fact otherwise it would produce generic Yang-Mills moduli spaces over $X \backslash p t$ as smooth manifolds. On the other hand, detailed analysis on the moduli spaces verified that it was impossible for such spaces to exist. Thus the smooth structure of the end was sufficiently complicated to obtain uncountably many exotic $\mathbb{R}^{4} \mathrm{~s}$. 


\section{B Casson handles}

Casson handles $(\mathrm{CH})$ can be constructed inside smooth four-manifolds. One defines a smooth structure on $\mathrm{CH}$ by restriction. In another words, a tower consists of smoothly-immersed two-handles, and so the tower itself admits a smooth structure. Each building block by an immersed two handle is called a kinky handle. Even though any Casson handle is homeomorphic to the standard open two-handle, many of them are not diffeomorphic to the standard one. In fact even for the simplest Casson handle, $\mathrm{CH}_{+}$, consisting of a single and positive kink at each stage, the following is known:

Lemma 0.1 [4, 5] $\mathrm{CH}_{+}$is exotic in the sense that the attaching circle cannot bound a smoothly embedded disc inside $\mathrm{CH}_{+}$.

For each signed infinite tree there corresponds a $\mathrm{CH}$ as a smooth four-manifold. In this paper we will define a subclass of signed infinite trees which are called homogeneous trees of bounded type. These are constructed iteratively by attaching infinitely many half-periodic trees. A connected infinite subtree of a homogeneous tree of bounded type is called a tree of bounded type. Notice that a Casson handle of a tree of bounded type admits a smooth embedding by another $\mathrm{CH}$ of a tree of homogeneously bounded type. In this paper we will use the term Casson handle of bounded type to refer to any Casson handle constructed from a signed tree of homogeneously bounded type.

Now any Casson handle of bounded type can be smoothly embedded in $\mathrm{CH}_{+}$ preserving the attaching circles. The above immediately implies the following:

Corollary 0.1 Any $\mathrm{CH}$ of bounded type is exotic.

Typically, smooth structures on Casson handles will have a deep effect on the smooth types of four-manifolds which contain them. Thus Casson handles are smooth open four-manifolds with the attaching region, and comprise a rich class among open four-manifolds. They will provide highly nontrivial examples to study in open manifold theory. It is difficult to understand the important numerical relationship between the growth of signed trees and the complexity of smooth structures on the corresponding Casson handles. In [18] some relation between Stein structures and the number of kinks was found. 


\section{C Outline of the article}

In this paper we study Yang-Mills gauge theory over Casson handles. Our final aim will be to measure the complexity of smooth structures on Casson handles by means of gauge theory. Roughly speaking in order to construct Yang-Mills theory on open four-manifolds, one needs to overcome two steps. The first is the Fredholm theory of the linearized equation. The other is perturbation theory, or transversality, where the setting of perturbation is different from the closed case (as we will explain below). A general procedure in Yang-Mills theory tells us that in a situation on four-manifolds where the Fredholm theory is applicable, one can obtain Yang-Mills moduli spaces as generically smooth manifolds of finite dimension. In order to induce information on smooth fourmanifolds from these moduli spaces, they are required to be non-empty under generic perturbation, where transversality theory will enter effectively.

In this paper we show that for Casson handles of bounded type, one can impose complete Riemannian metrics and weight functions on them so that Fredholm theory can be done. So we will obtain Yang-Mills moduli spaces on them as generically smooth manifolds of finite dimension. Perturbation theory on Casson handles is at present under development.

Let $(S, g)$ be a Riemannian (possibly open) oriented four-manifold, and $E \rightarrow S$ be an $S O(3)$ bundle. A connection $A$ on $E$ is called anti-self-dual $(A S D)$ if its curvature form satisfies the equation $F_{A}^{+} \equiv F_{A}+* F_{A}=0$. Roughly speaking when $S$ is closed, the set of ASD connections modulo the gauge group is the Yang-Mills moduli space, which is generically a smooth manifold of finite dimension. In this case one does not have to take care so much on the underlying function spaces. Any choice among various Sobolev spaces $W^{k}(S)$ ( $k$ large) gives the same moduli space.

When $S$ is open, there are no standard choices of function spaces. Let $w: S \rightarrow$ $[0, \infty)$ be a smooth function. Then one obtains weighted Sobolev spaces $W_{w}^{k}(S)$. Let us choose an ASD connection $A_{0}$, and consider two sets. One is $\widetilde{\mathfrak{M}}$, the set of ASD connections such that their curvature forms are in $L^{2}$. The other is $\widetilde{\mathfrak{M}}\left(A_{0}\right)=\left\{A: A\right.$ is $\left.\mathrm{ASD}, A-A_{0} \in W_{w}^{k}\right\}$. In general these spaces are very different and it seems difficult to study the geometry of the former space. In a periodic case, one can see that these give the same moduli space modulo gauge transformation, which follows from the exponential decay estimate on the curvature forms. A standard argument on transversality also works using the decay estimate. For the case of Casson handles, it seems natural to regard these spaces $\widetilde{\mathfrak{M}}$ and $\widetilde{\mathfrak{M}}\left(A_{0}\right)$ as having mutually different natures. The exoticness of 
smooth types of various Casson handles come from how the boundary solid tori are attached. These data are put in the cylindrical direction in our choice of metric. So it would be a delicate matter how these moduli spaces behave near the ends. The complexity of these moduli spaces will reflect that of the Casson handles.

In our class of Casson handles of bounded type, we will show that a Fredholm theory can be constructed using $\widetilde{\mathfrak{M}}\left(A_{0}\right)$. Our main concern here is analysis of the Atiyah-Hitchin-Singer complex:

$$
0 \longrightarrow W_{w}^{k+1}((Y, g)) \stackrel{d}{\longrightarrow} W_{w}^{k}\left((Y, g) ; \Lambda^{1}\right) \stackrel{d^{+}}{\longrightarrow} W_{w}^{k-1}\left((Y, g) ; \Lambda_{+}^{2}\right) \longrightarrow 0 .
$$

In this paper we will explicitly construct Riemannian metrics and weight functions over Casson handles, so that the above complex is Fredholm and its cohomology groups are calculable when restricted to our class. This is the Fredholm part which we mentioned above. One may generalize this to the case with coefficients in the adjoint bundle $\operatorname{Ad}(\mathfrak{g})$ of $E$. We denote by $H_{A^{\prime}}^{*}(\mathrm{AHS})$ the cohomology groups with coefficient at $A^{\prime} \in \mathfrak{M}$.

Suppose the above complex is Fredholm. Then roughly speaking the ASD moduli space $\widetilde{\mathfrak{M}}\left(A_{0}\right) / \mathfrak{G}$ has a local model $H_{A^{\prime}}^{1}=\operatorname{ker} d_{A^{\prime}}^{+} / \operatorname{im} d_{A^{\prime}}$ at $A^{\prime}$. In fact the moduli space has the structure of a finite-dimensional manifold at $A^{\prime}$, when $d_{A^{\prime}}$ is injective and $d_{A^{\prime}}^{+}$is surjective. In that case $H_{A^{\prime}}^{1}$ is canonically isomorphic to the tangent space of the moduli space at $A^{\prime}$. These properties are well known for closed four-manifolds. A parallel argument also works for the open case. Once one fixes a 'base' $L^{2}$ ASD connection $A_{0} \in \mathfrak{M}$, then transversality also works for this case. Thus if one takes a generic metric $g^{\prime}$ with respect to $A_{0}$, then the corresponding moduli space $\widetilde{\mathfrak{M}}\left(A_{0}, g^{\prime}, w\right) / \mathfrak{G}$ will have the structure of a finite-dimensional manifold. Notice that these moduli spaces are parameterized by $A_{0} \in \mathfrak{M}$, and $g^{\prime}$ will depend on $A_{0}$. This causes some difficulty in perturbation theory.

\section{D Main results}

Here is the main theorem:

Theorem 0.1 Let $S$ be a smooth oriented open four-manifold constructed by attaching to a zero handle Casson handles of homogeneous trees of bounded type. Then there exists a complete Riemannian metric of bounded geometry $g$ and an weight function $w$ on $S$ such that one can construct ASD moduli spaces over $S$ as finite dimensional smooth manifolds with respect to $(S, g, w)$. 
This follows from the next two propositions. In this paper we will introduce admissibility for a pair of a Riemannian metric and a weight function over an open four-manifold (1.A). Then we will show the following:

Proposition 0.1 Let $S$ be an open four-manifold. Suppose one can equip an admissible pair $(g, w)$ over $S$. Then one can construct $A S D$ moduli spaces over $S$ as finite-dimensional smooth manifolds.

In order to obtain admissible pairs over Casson handles of bounded type, we will use an iterative method. The main construction in this paper is the following:

Proposition 0.2 Let $S$ be in theorem 0.1. There exist a complete Riemannian metric and an weight function on $S$ so that the corresponding AtiyahHitchin-Singer (AHS) complex over $S$ becomes Fredholm. Moreover the metric and the weight function over $S$ give an admissible pair.

Let us outline the construction of Riemannian metrics on Casson handles by restricting to simple cases. Each building block of a Casson handle, namely a kinky handle, is diffeomorphic to $\llbracket\left(S^{1} \times D^{3}\right)$ with two attaching regions; one is a tubular neighborhood of band sums of Whitehead links (this is connected with the previous block), and the other is a disjoint union of the standard open subsets $S^{1} \times D^{2}$ in $\sharp S^{1} \times S^{2}=\partial\left(\llbracket S^{1} \times D^{3}\right)$ (this is connected with the next block). The number of end-connected sums is exactly the number of selfintersections of the immersed two handle. The simplest Casson handles have $S^{1} \times D^{3}$ as their building blocks. We attach a Casson handle to the zero-handle along the attaching circle and denote it by $S=D^{4} \cup C H$.

Let us consider a simple Casson handle, say $C H\left(\mathbb{R}_{+}\right)$, a periodic Casson handle by positive kinks. Unlike the Taubes construction, the building blocks here are open. In order to make end-connected sums of building blocks isometrically, one explicitly equips the metrics on the building blocks. Then the building block as an open manifold becomes an 'open cylindrical' manifold. As in the Taubes construction, one connects two attaching regions in a block. The result becomes a cylindrical manifold on which analysis is already well known. By equipping it with a suitable weight function, one will apply the Fourier-Laplace transform between the cylindrical manifold and its periodic cover. By a kind of excision, one obtains a Fredholm AHS complex over $S$.

This method shows that once one obtains some suitable function spaces on any open manifolds, then the Fourier-Laplace transform works on their periodic covers. We will use this observation iteratively. In general a Casson handle can 
be expressed by an infinite tree with one end point and with a sign \pm on each edge. The next simplest Casson handle will be represented as follows. Let $\mathbb{R}_{+}$ be the half-line with the vertices $\{0,1,2, \ldots\}$. We prepare another family of half-lines $\left\{\mathbb{R}_{+}^{i}\right\}_{i=1,2, \ldots}$ assigned with indices. Then we obtain another infinite tree:

$$
R(2)=\mathbb{R}_{+} \cup_{i=1,2, \ldots} \mathbb{R}_{+}^{i}
$$

where we connect $i$ in $\mathbb{R}_{+}$with 0 in $\mathbb{R}_{+}^{i}$. For example one may assign - on $\mathbb{R}_{+}$and + on all $\left\{\mathbb{R}_{+}^{i}\right\}_{i}$. Then one obtains the corresponding Casson handle $C H(R(2))$. In this case the building blocks are diffeomorphic to $\bar{\Sigma}_{2}=\left(S^{1} \times\right.$ $\left.D^{3}\right) \mathfrak{\natural}\left(S^{1} \times D^{3}\right)$ along $\mathbb{R}_{+} \cdot \bar{\Sigma}_{2}$ has three attaching components. One is $\mu$, the tubular neighborhood of the band sum of two Whitehead links as before. We will denote the others by $\mu^{\prime}$ and $\gamma$, where these represent a generator of $\pi_{1}\left(\bar{\Sigma}_{2}\right)$. In order to apply Fourier-Laplace transform, one takes end-connected sums twice. Firstly one takes the end-connected sum between $\mu$ and $\mu^{\prime}$ as before. The result is an 'open cylindrical' manifold, since there still remains one attaching region, $\gamma$. One takes the end-connected sum of this with $C H\left(\mathbb{R}_{+}\right)$along $\gamma$. In this manner, one obtains another open manifold, $\left(\bar{\Sigma}_{2} / \mu \sim \mu^{\prime}\right) \bigsqcup_{\gamma} C H\left(\mathbb{R}_{+}\right)$. Now we have already two kinds of analytic preparations. One is analysis for cylindrical manifolds, and the other for the half-periodic Casson handle as we have explained. By a kind of excision argument, one can verify that the AHS complex on the open manifold is Fredholm. Half of part of its periodic covering is exactly $C H(R(2))$. Again by a Fourier-Laplace transform and excision, one obtains the Fredholm AHS complex over $S=D^{4} \cup C H(R(2))$.

These are simple examples, but the idea works for much more general cases of Casson handles. One may iterate this construction inductively to more complicated Casson handles.

In the case of end-connected sums, the excision argument tells us that just the differentials have closed range. In order to see the finite-dimensionality of the cohomology, we will make explicit calculations. This is one point where we use de Rham cohomology calculations. On this point a parallel argument by Seiberg-Witten theory seems to have some technical difficulty.

\section{E Directions for further research}

Finally let us indicate some possible developments arising from this kind of analysis, assuming perturbation theory. We would like to propose here some problems on the study of smooth structures on Casson handles. 
Let us consider algebraic surfaces, say the K3 surface. This decomposes topologically as $2\left|-E_{8}\right| \sharp 3\left(S^{2} \times S^{2}\right)$, and contains six Casson handles. One may guess that it would be impossible to do Yang-Mills gauge theory on Casson handles inside the K3 surface, or more generally inside many of algebraic surfaces. One can verify that at least one of Fredholm theory or perturbation theory breaks down 21]. It seems reasonable to think that the smooth structure on the Casson handles in K3 will be so complicated that one might not be able to do Fredholm theory. If perturbation theory could work on these, then one will be able to tell that homogeneous Casson handles inside K3 should grow more than exponentially. The argument is to construct Yang-Mills theory on Casson handles and it will lead to a contradiction by dimension-counting on the moduli spaces.

Let us take two Casson handles, $C H\left(T_{1}\right)$ and $C H\left(T_{2}\right)$ where $T_{i}$ are the corresponding signed trees. When $T_{1}$ is embedded into $T_{2}$, then there is a smooth embedding, $\mathrm{CH}\left(\mathrm{T}_{2}\right) \hookrightarrow C H\left(T_{1}\right)$ preserving the attaching circles, but one cannot say about converse embeddings of $C H\left(T_{1}\right)$ into $C H\left(T_{2}\right)$ in general. The above argument suggests that any $\mathrm{CH}$ of bounded type will not be able to embed into Casson handles inside K3 preserving the attaching circles.

In our situation here, one treats Casson handles whose trees grow polynomially. In fact in our method, one might expect that as Casson handles grow near exponentially, the continuous spectrum with respect to the AHS complex will approach zero. It might be possible that even exponential growth is already too complicated to obtain Fredholm theory. On the other hand one does not know concretely how the signed trees grow for the case of Casson handles in K3 (for this direction, see [3]). In reality, any Casson handles of bounded type can appear in $S^{2} \times S^{2} \backslash p t$, and so it would be interesting to study smooth types on $S^{2} \times S^{2} \backslash p t$ arising from Casson handles of bounded type.

Next we will consider another problem. The re-imbedding theorem gives another Casson handle inside a six stage tower preserving the attaching circles. Now any Casson handle $C H$ of bounded type can be embedded into one of the simplest Casson handles $C H_{ \pm} \equiv C H\left(\mathbb{R}_{ \pm}\right)$, say into $\mathrm{CH}_{+}$. Let $C H_{+}(n)$ be the $n$th stage of $\mathrm{CH}_{+}$. One may consider a question whether $\mathrm{CH}$ can be smoothly embedded into $\mathrm{CH}_{+}(n)$ for some large $n$ preserving the attaching circles. For this, we would like to outline a possible argument (see [5]). Let $\left(Z, \partial_{0} Z, \partial_{1} Z\right)$ be a smoothly non-product $h$-cobordism with different Donaldson's polynomials on two boundaries. By using Kirby calculus technique, one may find a decomposition $Z=W \cup U$ where $W$ is smoothly product and $C H_{+}(n)$ appears in both the ends, $\partial_{0} Z \cap W$ and $\partial_{1} Z \cap W$. If $C H$ could be smoothly embedded into $\mathrm{CH}_{+}(n)$, then one will obtain Yang-Mills moduli spaces over both of $\partial_{i} Z \cap W$ 
whose ends are consisted by $\mathrm{CH}$ of bounded type. Since these are diffeomorphic, the Donaldson's invariants over them will have the same numerical value, which would contradict the assumption. In the above situation, one would be able to conclude that there are no smooth embeddings of $\mathrm{CH}$ into any finite-stage approximations $\mathrm{CH}_{+}(n)$ preserving the attaching circles.

The author would like to express his gratitude to the Max Planck Institut for their hospitality, during his visit. He also would like to express his appreciation of the referees for numerous comments and suggestions.

\section{$1 \quad$ Yang-Mills moduli space}

\section{A Admissible four-manifolds}

In this section, one will construct Yang-Mills moduli spaces over non compact four-manifolds. We treat open four-manifolds which can admit Riemannian metrics and weight functions such that they are able to construct a Fredholm complex.

Let $Y$ be a non compact smooth four-manifold. Throughout this section, one always assumes that $Y$ is simply-connected, and simply-connected at infinity.

Let $g$ be a complete Riemannian manifold of bounded geometry and $w: Y \rightarrow$ $[0, \infty)$ be a smooth function. Recall that a complete Riemannian metric is of bounded geometry, if (1) the injectivity radius is more than a positive constant $\epsilon>0$ at any point, and (2) for any $l \geq 0$, $\sup _{x \in Y}\left|\nabla^{l} R\right|<\infty$, where $\nabla$ and $R$ are with respect to $g$. Throughout this paper, we always assume that $g$ is of bounded geometry, and $\left|\nabla^{l}(w)\right| C^{0}(Y)<\infty$ for all $l \geq 1$.

Let $\Lambda^{i}(Y)$ and $\Lambda_{+}^{2}(Y)$ be the exterior differentials on $i$-forms and self dual 2 -forms with respect to $g, i=0,1$. Then we have the Atiyah-Hitchin-Singer complex (without coefficient) as:

$$
0 \longrightarrow C_{c}^{\infty}\left(Y ; \Lambda^{0}\right) \stackrel{d}{\longrightarrow} C_{c}^{\infty}\left(Y ; \Lambda^{1}\right) \stackrel{d^{+}}{\longrightarrow} C_{c}^{\infty}\left(Y ; \Lambda_{+}^{2}\right) \longrightarrow 0
$$

where $d^{+}=(1+*) \circ d$. Using the $L^{2}$ adjoint operator (here we do not use any weight functions), we get the next elliptic operator:

$$
P=d^{+} \oplus d^{*}: C_{c}^{\infty}\left(Y ; \Lambda^{1}\right) \rightarrow C_{c}^{\infty}\left(Y ; \Lambda^{0} \oplus \Lambda_{+}^{2}\right) .
$$

Let us introduce weighted Sobolev $k$-norms on $Y$ by:

$$
|u| W_{w}^{k}=\left(\Sigma_{l \leq k} \int \exp (w)\left|\nabla^{l} u\right|^{2} v o l\right)^{\frac{1}{2}}
$$


We denote these Sobolev weighted spaces by $W_{w}^{k}$ or $L_{w}^{2}($ for $k=0)$. Then one has a complex of bounded maps:

$$
0 \longrightarrow W_{w}^{k+2}\left(Y ; \Lambda^{0}\right) \stackrel{d}{\longrightarrow} W_{w}^{k+1}\left(Y ; \Lambda^{1}\right) \stackrel{d^{+}}{\longrightarrow} W_{w}^{k}\left(Y ; \Lambda_{+}^{2}\right) \longrightarrow 0
$$

Now let us say that the pair $(g, w)$ is admissible, if the following conditions are satisfied:

(1) The above consists of a Fredholm complex, namely each differential has closed range, and its cohomology group is of finite dimension as a vector space over $\mathbb{R}$. We denote their cohomology groups by $H^{*}(\mathrm{AHS}), *=0,1,2$. Notice that by the condition, one has $L_{w}^{2}$ adjoint operators $d_{w}^{*}$ and $\left(d_{+}\right)_{w}^{*}$.

(2) $Y$ admits a partition $Y=K \cup Y_{1} \cup \ldots Y_{l}$ such that (a) $K$ is a compact subset and each $Y_{j}$ is an open subset of $Y$, and (b) let us put $D=d_{w}^{*} \oplus d_{+}$ or $d \oplus\left(d_{+}\right)_{w}^{*}$. Then there is a positive constant $C_{k}$ such that for any $u \in$ $C_{c}^{\infty}\left(Y_{j} ; \Lambda^{*}\right), *=1,2$, one has the bound $|u| W_{w}^{k+1} \leq C|D(u)| W_{w}^{k}$.

(3) For any $f \in C^{1}(Y)$ with $|d f| L_{w}^{2}(Y)<\infty$, there is $\bar{f} \in \mathbb{R}$ with $f-\bar{f} \in$ $L_{w}^{2}(Y)$.

(4) There is a compact subset $K \subset Y$ and a homeomorphism $Y \backslash K \cong S^{3} \times$ $[0, \infty)$.

In sections 2 to 5 , we will construct admissible pairs for all Casson handles constructed from homogeneous signed trees of bounded type.

Proposition 1.1 Let $(g, w)$ be an admissible pair on $Y$. Then $H^{0}(\mathrm{AHS})=$ $H^{1}(\mathrm{AHS})=0$ and $H^{2}(\mathrm{AHS}) \geq k=H_{+}^{2}(Y ; \mathbb{R})$.

Proof Clearly $H^{0}(\mathrm{AHS})=H^{1}(\mathrm{AHS})=0$ by the admissibility condition (3) . We show $\operatorname{dim} H^{2}$ (AHS) $\geq k$. For this, one takes two steps.

Let $H_{\mathrm{cp}}^{2}(Y ; \mathbb{R})^{+}$be a subspace of $H_{\mathrm{cp}}^{2}(Y ; \mathbb{R})$ consisting of vectors with $\int_{Y} u \wedge u>$ 0 . Then $H_{\mathrm{cp}}^{2}(Y ; \mathbb{R})^{+}$is a linear subspace of $H^{2}(Y ; \mathbb{R})$ of dimension $k$, since the natural map $I: H_{\mathrm{cp}}^{2}(Y ; \mathbb{R}) \rightarrow H^{2}(Y ; \mathbb{R})$ gives an isomorphism.

Now by the above, one has $\operatorname{dim} H_{\mathrm{cp}}^{2}(Y ; \mathbb{R})^{+}=k$. For any element $u \in$ $C^{\infty}\left(Y ; \Lambda^{2}\right)$, let us denote by $u^{+}$the projection to $\Lambda_{+}^{2}$ part. Then one defines:

$$
p: H_{\mathrm{cp}}^{2}(Y ; \mathbb{R})^{+} \rightarrow H^{2}(\mathrm{AHS})
$$

by assigning $[u] \mapsto\left[u^{+}\right]$. This map is well defined. We show that $p$ is an injection.

Suppose $\left[u^{+}\right]=0 \in H^{2}$ (AHS). Then one has $\alpha \in W_{w}^{1}\left(Y ; \Lambda^{1}\right)$ such that $u^{+}=d^{+}(\alpha)$, or $(u-d(\alpha))^{+}=0$. Let us put $v=u-d(\alpha)$, and take the cup 
product $\langle v, v\rangle=\int_{Y} v \wedge v$. One can use the Stokes theorem to see $\int_{Y} u \wedge d(\alpha)=$ $\int_{Y} d(u \wedge \alpha)=0$ ( $u$ is a closed form). Thus one has the equality $\int_{Y} v \wedge v=$ $\int_{Y} u \wedge u \geq 0$. On the other hand since $v$ does not have self-dual part, one has

$\int_{Y} v \wedge v \leq 0$. This shows $u=d(\alpha)$. This implies $[u]=0 \in H_{\mathrm{cp}}^{2}(Y ; \mathbb{R})^{+}$since $I$ gives an isomorphism. Thus one concludes $\operatorname{dim} H^{2} \geq k$.

Example 1.1 In general, $\operatorname{dim} H_{c p}^{2}(Y ; \mathbb{R})^{+}$and $\operatorname{dim} H^{2}(\mathrm{AHS})=\operatorname{ker}\left(d_{+}\right)_{w}^{*}$ do not coincide. One takes $Y=\mathbb{R}^{2} \times \mathbb{R}^{2}$, where one equips the standard metric on $\mathbb{R}^{2}$, and the product one on $Y$. Let us choose a weight function $w$ on $Y$. We show $\operatorname{dim} H^{2}$ (AHS) is nonzero. Let $u_{1}$ and $u_{2}$ be 2 -forms on $\mathbb{R}^{2}$ respectively, such that $\left|u_{i}\right| \equiv 1, i=1,2$, pointwisely. Then clearly $d u_{i}=0, i=1,2$, and $u_{1}+u_{2}$ gives a self-dual 2 -form with bounded pointwise norm. Then one puts $v=\exp (-w)\left(u_{1}+u_{2}\right) \in L_{w}^{2}\left(Y ; \Lambda_{+}^{2}\right)$. Moreover $v$ satisfies the equation $d(\exp (w) v)=0$. Thus $v$ is a nontrivial element in $H^{2}$ (AHS).

\section{B ASD moduli space}

Let $E \rightarrow Y$ be a $G$-vector bundle (where $G=S O(3)$ or $U(1)$ ) such that except on some compact subset $K \subset Y, E \mid Y \backslash K$ is trivial. One denotes the corresponding principal $G$-bundle by $P$. For $S O(3)$-bundles, $E$ is determined by $w_{2}(E) \in H^{2}\left(Y ; \mathbb{Z}_{2}\right)$. In later sections, we fix a trivialization of $E \mid Y \backslash K$. Thus we fix an $S O(3)$-bundle with $w_{2}$ and $p_{1}(E)$. Let $A$ be a smooth connection over $E$ such that except some compact subset on $Y$, it satisfies the ASD equation:

$$
F_{A}+* F_{A}=0
$$

where $F_{A}$ is the curvature form of $A$. Let us denote by $\widetilde{R}(Y)$ the set of smooth connections as above satisfying $\left|F_{A}\right| L^{2}(Y)<\infty$. By changing a trivialization, one may assume $|a| L^{2}(Y \backslash K)<\infty$ where $A=d+a$ on $Y \backslash K$. Later we always assume this property. If $A$ is the trivial connection except some compact subset, then it is an element in $\widetilde{R}(Y)$.

Lemma 1.1 For $A \in \widetilde{R}(Y), p_{1}(A)=\frac{1}{4 \pi^{2}} \int_{Y} \operatorname{tr}\left(F_{A} \wedge F_{A}\right)$ is an integer.

In order to show this, one uses the following:

Sublemma 1.1 14] Let $D \subset Y$ be a $\delta$-ball with any point as the center, where $\delta>0$ is sufficiently small. Then there exists another small $\epsilon>0$ with 
the following property; suppose $\left|F_{A}\right| L^{2}(D)<\epsilon$. Then there exists a gauge transformation $g$ over $D$, such that:

$$
\sup _{x \in D}\left|\nabla^{l}\left(g^{*}(A)-d\right)\right|(x) \leq C_{l}\left|F_{A}\right| L^{2}(D)
$$

where $C_{l}$ are constants, independent of $A$.

Proof of lemma Let us take any small $\epsilon>0$. Then there exists a compact subset $K \subset Y$, and a smooth gauge transformation $g \in C^{\infty}(Y$; $\operatorname{Aut}(E))$ such that $\left|g^{*} A-d\right| W^{1}(Y \backslash K)<\epsilon$, where $d$ is the trivial connection ( $Y$ is simplyconnected at infinity). In order to get $g$, one needs to use the above sublemma and the patching argument. We omit to describe the process (see [11]). One may assume that $K$ is a compact submanifold with boundary. Let $D(K)$ be a double of $K$. Then using a cut-off function $\varphi$ around $\partial K$, one may extend $A^{\prime} \equiv \varphi\left(g^{*} A\right)$ over $D(K)$, by putting $d$ over the other side of $K \subset D(K)$. By the Chern-Weil theory, $p_{1}\left(A^{\prime}\right)$ is an integer. On the other hand, since $p_{1}(A)-p_{1}\left(A^{\prime}\right)$ may be arbitrarily small as above, one concludes that $p_{1}(A)$ is an integer. This completes the proof.

Let us put $\operatorname{Ad}(P)=P \times_{G} \mathfrak{G}$, where $\mathfrak{G}$ is the Lie algebra of $G .\left(P \times_{G} \mathbb{R}^{3}=E\right)$. We also denote by $\mathfrak{G}$ the trivial Lie $G$-bundle. Then we have the AtiyahHitchin-Singer complex (AHS complex) as:

$$
0 \longrightarrow C_{c}^{\infty}(Y ; \operatorname{Ad}(P)) \stackrel{d_{A}}{\longrightarrow} C_{c}^{\infty}\left(Y ; \operatorname{Ad}(P) \otimes \Lambda^{1}\right) \stackrel{d_{A}^{+}}{\longrightarrow} C_{c}^{\infty}\left(Y ; \operatorname{Ad}(P) \otimes \Lambda_{+}^{2}\right) \longrightarrow 0
$$

where $d_{A}^{+}=(1+*) \circ d_{A}$. Using the $L_{w}^{2}$ adjoint operator, we get the next elliptic operator:

$$
P(A)_{w}=\left(d_{A}\right)_{w}^{*} \oplus d_{A}^{+}: W_{w}^{k+1}\left(Y ; \operatorname{Ad}(P) \otimes \Lambda^{1}\right) \rightarrow W_{w}^{k}\left(Y ; \operatorname{Ad}(P) \otimes\left(\Lambda^{0} \oplus \Lambda_{+}^{2}\right)\right) .
$$

Let us fix $A_{0} \in \widetilde{R}(Y)$. One defines an affine Hilbert space as:

$$
\mathfrak{A}_{k}(P)_{0}=\left\{A_{0}+a \mid a \in W_{w}^{k}\left(Y ; \operatorname{Ad} P \otimes T^{*} Y\right)\right\}, \quad k \geq 3 .
$$

Let us take $g \in C_{\text {loc }}^{1}(Y$; Aut $E)$. By embedding as $g \in C_{\text {loc }}^{1}(Y ; \operatorname{Hom}(E, E))$, one may consider $\nabla_{A_{0}} g \in C^{0}\left(Y ; \operatorname{Hom}(E, E) \otimes T^{*} Y\right)$. Notice that if $g$ is locally $W^{4}$, then it is of $C^{1}$ class. Now one defines the weighted Sobolev gauge group:

$$
\begin{aligned}
& \mathfrak{G}_{l}(P)=\left\{h \in W_{l o c}^{l}(Y ; \operatorname{Aut}(E)) \mid \nabla_{A_{0}} h \in W_{w}^{l-1}\left(Y ; \operatorname{Hom}(E, E) \otimes T^{*} Y\right)\right\}, \quad l \geq 4 . \\
& \mathfrak{G}_{l}(P)_{0}=\left\{h \in W_{l o c}^{l}(Y ; \operatorname{Aut}(E)) \mid h-i d \in W_{w}^{l-1}\left(Y ; \operatorname{Hom}(E, E) \otimes T^{*} Y\right)\right\} .
\end{aligned}
$$

First of all one has the following property of $\mathfrak{G}_{l}(P)$. Suppose one of the following two conditions; (1) $G=U(1)$, or (2) $G=S O(3)$ and $A \mid Y \backslash K \in$ $W_{w}^{k}\left(Y \backslash K ; \operatorname{Ad}(P) \otimes \Lambda^{1}\right)$ where the trivialization of $E \mid Y \backslash K$ is fixed. Then: 
Lemma 1.2 For each $h \in \mathfrak{G}_{k+1}(P)$, there exists $\bar{h} \in G$ such that $h-\bar{h} \in$ $W_{w}^{k}(Y \backslash K ; \operatorname{Aut}(E))$, where $\bar{h}$ is a constant gauge transformation.

Proof This follows from Kato's inequality, $\left|\nabla_{A_{0}} h\right| \geq d|h|$ almost everywhere, and admissibility condition (3).

The Lie algebras of $\mathfrak{G}_{l}(P)$ and $\mathfrak{G}_{l}(P)_{0}$ are correspondingly as follows:

$$
\begin{aligned}
& \mathfrak{g}_{l}(P)=\left\{h \in W_{l o c}^{l}(Y ; \operatorname{Ad}(P)) \mid \nabla_{A_{0}} h \in W_{w}^{l-1}\left(Y ; \operatorname{Ad}(P) \otimes T^{*} Y\right)\right\}, \\
& \mathfrak{g}_{l}(P)_{0}=W_{w}^{l}(Y ; \operatorname{Ad}(P)) .
\end{aligned}
$$

Lemma 1.3 (1) $\mathfrak{G}_{k+1}(P)_{0}$ acts on $\mathfrak{A}_{k}(P)_{0}$ by $g^{*}\left(A_{0}+a\right)=g^{-1} \nabla_{A_{0}} g+g^{-1} a g$.

(2) Suppose $P$ is a $U(1)$-bundle with $G=U(1)$. Then $\mathfrak{G}_{k+1}(P)$ acts on $\mathfrak{A}_{k}(P)$.

The proof is standard, and we omit it.

Notice that $\mathfrak{G}_{k+1}(P)$ may not act on $\mathfrak{A}_{k}(P)_{0}$, since $A_{0}$ may live only in $L^{2}(Y)$.

Let us define:

$$
\widehat{\mathfrak{M}}_{k}(P)=\left\{A \in \mathfrak{A}_{k}(P)_{0}: F_{A}+* F_{A}=0\right\} .
$$

Then $\mathfrak{G}_{k+1}(P)_{0}$ acts on $\widehat{\mathfrak{M}}_{k}(P)$. Thus one gets the quotient space:

$$
\mathfrak{M}_{k}(P)=\widehat{\mathfrak{M}}_{k}(P) / \mathfrak{G}_{k+1}(P)_{0} .
$$

Remark 1.1 $\mathfrak{M}_{k}(P)$ is constructed after choosing a base connection $A_{0} \in$ $\widetilde{R}(Y)$ and $W_{w}^{k}$ Hilbert spaces. It can be shown that this space is in fact independent of choice of $k$. However it would definitely depend on choice of $A_{0}\left(A_{0}\right.$ lies only in $L^{2}$ with respect to the trivialization on the end). Thus one could express the space as:

$$
\mathfrak{M}_{k}(P)=\mathfrak{M}_{k}\left(P, A_{0}\right) \text {. }
$$

Let us find a linear space which corresponds to the tangent space of $\mathfrak{M}(P)_{k}$.

Lemma 1.4 There exist natural continuous maps:

$$
\exp : \mathfrak{g}_{k+1}(P), \quad\left(W_{w}^{k+1}(Y ; \operatorname{Ad}(P))\right) \rightarrow \mathfrak{G}_{k+1}(P) \quad\left(\mathfrak{G}_{k+1}(P)_{0}\right) .
$$

Moreover, let us put $\mathfrak{G}_{k+1}^{\prime}(P)(\epsilon)=\left\{g \in \mathfrak{G}_{k+1}(P) \| g-1 \mid W_{w}^{k+1}(Y) \leq \epsilon\right\}$. Then for sufficiently small $\epsilon>0$, there exists $\log : \mathfrak{G}_{k+1}(P)_{0}(\epsilon) \rightarrow W_{w}^{k+1}(Y ; \operatorname{Ad} P)$ which inverts exp. One has a similar statement for $\exp \mid W_{w}^{k+1}(Y ; \operatorname{Ad}(P))$. 
We also omit the proof.

Let us take any $A \in \mathfrak{A}_{k}(P)_{0}$, and consider the following continuous map:

$$
d_{A}: \mathfrak{g}_{k+1}(P) \rightarrow W_{w}^{k}\left(Y ; \operatorname{Ad} P \otimes T^{*} Y\right)
$$

by $\left.u \rightarrow \frac{d}{d t}(\exp (t u) A)\right|_{t=0}$. Locally this map is expressed as $u \rightarrow d u+[u, a]$.

Lemma 1.5 Suppose an admissible pair $(g, w)$ is given. Then for the AHS complex, $d_{A}$ and $d_{A}^{+}$are closed operators.

Proof Let us show that $d_{A} \mid W_{w}^{k+1}(Y ; \operatorname{Ad}(P))$ has closed range. Let $A_{0}^{\prime} \in$ $\mathfrak{A}_{k}(P)$ be a smooth connection which coincides with $A$ on some compact subset $K$, and is trivial except on another compact subset. Then one has $A=A_{0}^{\prime}+a$, $a \in W^{k}\left(Y ; \operatorname{Ad} P \otimes T^{*} Y\right)$. Let $\varphi$ be a cut-off function such that for some compact subsets $K \subset K^{\prime} \subset K^{\prime \prime} \subset Y$, one has $\varphi \mid K^{\prime} \equiv 1$ and $\varphi \mid\left(K^{\prime \prime}\right)^{c} \equiv 0$. By the assumption, $d: W_{w}^{k+1}\left(Y ; \Lambda^{0}\right) \rightarrow W_{w}^{k}\left(Y ; \Lambda^{1}\right)$ is invertible (satisfies $|d(u)| W_{w}^{k} \geq$ $\left.C|u| W_{w}^{k+1}\right)$. Then one sees $d_{(1-\varphi) a}$ is invertible by choosing sufficiently large $K^{\prime} \subset K^{\prime \prime}$, using the Sobolev embedding, $W_{\mathrm{loc}}^{k} \hookrightarrow C^{0}$. Then one has the following estimate:

$$
\begin{aligned}
|u| W_{w}^{k+1} \leq & C\left(\left|d_{A}(u)\right| W_{w}^{k}+|u| W_{w}^{k}\right) \\
\leq & C\left(\left|d_{A}(u)\right| W_{w}^{k}+|\varphi u| W_{w}^{k}+|(1-\varphi) u| W_{w}^{k}\right) \\
\leq & C\left(\left|d_{A}(u)\right| W_{w}^{k}+|\varphi u| W_{w}^{k}+\left|d_{(1-\varphi) a}((1-\varphi) u)\right| W_{w}^{k}\right) \\
\leq & C\left(\left|d_{A}(u)\right| W_{w}^{k}+|\varphi u| W_{w}^{k}+\left|(1-\varphi) d_{(1-\varphi) a}(u)\right| W_{w}^{k}+\right. \\
& \left.\quad\left|\left[d_{(1-\varphi) a},(1-\varphi)\right] u\right| W_{w}^{k}\right) \\
\leq & C\left(\left|d_{A}(u)\right| W_{w}^{k}+|\varphi u| W_{w}^{k}+\left|(1-\varphi) d_{A}(u)\right| W_{w}^{k}+\right. \\
& \left.\quad\left|\left[d_{(1-\varphi) a},(1-\varphi)\right] u\right| W_{w}^{k}+\left|(1-\varphi)\left[\varphi a+A_{0}^{\prime}, u\right]\right| W_{w}^{k}\right) \\
\leq & C\left(\left|d_{A}(u)\right| W_{w}^{k}+|\psi u| W_{w}^{k}\right)
\end{aligned}
$$

where $\psi$ is another compactly-supported cut-off function with $\psi \mid \operatorname{Supp} \varphi \equiv 1$. The last inequality shows that $d_{A}$ is a closed operator (see lemma 3.1).

Next let us consider $d_{A}^{+}$. Notice that we have now the adjoint operator $\left(d_{A}\right)_{w}^{*}$. Then it is enough to see $d_{A}^{+}\left(\operatorname{ker}\left(d_{A}\right)_{w}^{*} \cap W_{w}^{k+1}\right) \subset W_{w}^{k}\left(Y ; \operatorname{Ad}(P) \otimes \Lambda_{+}^{2}\right)$ has closed range (see lemma 1.6 and lemma 3.1). Let us put $D=\left(d_{A}\right)_{w}^{*} \oplus d_{A}^{+}$. Then using admissibility condition (2) and the above argument, one gets a similar inequality $|u| W_{w}^{k+1} \leq C\left(|D(u)| W_{w}^{k}+|\psi u| W_{w}^{k}\right)$ where a cut-off function $\psi$ has compact support. In particular $D: W_{w}^{k+1} \rightarrow W_{w}^{k}$ has closed range. 
Let us take $u \in W_{w}^{k+1}\left(Y ; \operatorname{Ad}(P) \otimes \Lambda^{1}\right)$ with $\left(d_{A}\right)_{w}^{*}(u)=0$. Then by the above two facts, one has a similar inequality:

$$
|u| W_{w}^{k+1} \leq C\left(\left|d_{A}^{+}(u)\right| W_{w}^{k}+|\psi u| W_{w}^{k}\right) .
$$

This shows that $d_{A}^{+}$also has closed range. This completes the proof.

Corollary 1.1 Let $A \in \mathfrak{A}_{k}(P)_{0}$. Then the AHS complex

$$
\begin{aligned}
0 \longrightarrow W_{w}^{k+1}(Y ; \operatorname{Ad}(P)) \stackrel{d_{A}}{\longrightarrow} W_{w}^{k}\left(Y ; \operatorname{Ad}(P) \otimes \Lambda^{1}\right) \\
\quad \stackrel{d_{A}^{+}}{\longrightarrow} W_{w}^{k-1}\left(Y ; \operatorname{Ad}(P) \otimes \Lambda_{+}^{2}\right) \longrightarrow 0
\end{aligned}
$$

is a Fredholm complex of index $-2 p_{1}(P)+3\left(\operatorname{dim} H^{1}(\mathrm{AHS})-\operatorname{dim} H^{2}(\mathrm{AHS})\right)$.

The index computation uses the excision principle, or relative index theorem 20]. One can also use [2] and the method in section 5 here. One denotes their cohomology groups by $H_{A}^{*}(\mathrm{AHS}), *=0,1,2$.

Let us consider the restriction:

$$
d_{A}: W_{w}^{k+1}(Y ; \operatorname{Ad} P) \rightarrow W_{w}^{k}\left(Y ; \operatorname{Ad} P \otimes T^{*} Y\right) .
$$

By the above corollary, one may consider the adjoint operator:

$$
\left(d_{A}\right)_{w}^{*}: W_{w}^{k}\left(Y ; \operatorname{Ad} P \otimes T^{*} Y\right) \rightarrow W_{w}^{k-1}(Y ; \operatorname{Ad} P) .
$$

One understands $\left(d_{A}\right)_{w}^{*}$ in the geometric way as follows. Let us take $A \in$ $\mathfrak{A}_{k}(P)_{0}$. Then one has the 'tangent space' of $\mathfrak{A}_{k}(P)_{0} / \mathfrak{G}_{k+1}(P)_{0}$ at $[A]$ :

$$
\frac{W_{w}^{k}\left(Y ; \operatorname{Ad} P \otimes T^{*} Y\right)}{d_{A}\left(W_{w}^{k+1}(Y ; \operatorname{Ad} P)\right)} \cong \operatorname{ker}\left(d_{A}\right)_{w}^{*}: W_{w}^{k}\left(Y ; \operatorname{Ad} P \otimes T^{*} Y\right) \rightarrow W_{w}^{k-1}(Y ; \operatorname{Ad} P) .
$$

Let us take a smooth family of connections $A(t) \in \mathfrak{A}_{k}(P)_{0}, t \in[0,1]$. Then one has:

$$
\left.\frac{\partial F_{A(t)}^{+}}{\partial t}\right|_{t=0}=\left(d_{A(0)}+* d_{A(0)}\right) B \equiv d_{A(0)}^{+} B
$$

where $B=\left.\frac{d A(t)}{d t}\right|_{t=0} \in W_{w}^{k}\left(Y ; \operatorname{Ad} P \otimes T^{*} Y\right)$. Notice that near infinity, $F_{A(t)}$ can be written as $d A(t)+A(t) \wedge A(t)$. Each $A(t)$ can be written as $A(t)=A_{0}+a_{t}$, where $a_{t} \in W_{w}^{k}\left(Y ; \operatorname{Ad} P \otimes \Lambda^{1}\right)$. Since $F_{A_{0}}^{+}=0$, one sees that $F_{A_{t}}^{+}$is a smooth family in $W_{w}^{k-1}\left(Y ; \operatorname{Ad} P \otimes \Lambda_{+}^{2}\right)$. Now one gets the 'tangent space' of $\mathfrak{M}_{k}(P)$ at $[A]$ as:

$$
\operatorname{ker}\left(d_{A}\right)_{w}^{*} \oplus d_{A}^{+}: W_{w}^{k}\left(Y ; \operatorname{Ad} P \otimes T^{*} Y\right) \rightarrow W_{w}^{k-1}\left(Y ; \operatorname{Ad} P \otimes\left(\Lambda^{0} \oplus \Lambda_{+}^{2}\right)\right) .
$$

In particular this space is isomorphic to $H_{A}^{1}$ (AHS) which is of finite dimension. 
Lemma 1.6 $\operatorname{ker} d_{A}: W_{w}^{k+1}(Y ; \operatorname{Ad}(P)) \rightarrow W_{w}^{k}\left(Y ; \operatorname{Ad}(P) \otimes \Lambda^{1}\right)$ is zero.

Proof This follows from Kato's inequality, $\left|\nabla_{A} u\right| \geq|d| u||$ almost everywhere. Notice that there are also no $L^{2}$ functions $u$ with $d_{A}(u)=0$. This is also seen as follows: Suppose $u \in L^{2}(Y ; \operatorname{Ad}(P)),|u| L^{2}=1$, satisfies $d_{A}(u)=0$. Then for $g=\exp (u) \in \mathfrak{G}$, one has $g^{*}(A)=A$. Then it is known that for any path $l$ between $p$ and $q$ in $Y$, one has $P_{l} \circ g(p)=g(q) \circ P_{l}$, where $P_{l}$ is the parallel translation along $l$. Let $q \rightarrow \infty$. Then $g(q)$ approaches to the identity. On the other hand since $P_{l}$ holds the inner product, one has $\left\langle P_{l}(g(p)(u)), g(q)\left(P_{l}(u)\right)\right\rangle_{q} \sim\langle g(p)(u), u\rangle_{p}$. This shows that $g(p)$ is near the identity. This impossible. This completes the proof.

Let us consider $\mathfrak{M}_{k}(P)=\widehat{\mathfrak{M}}_{k}(P)_{0} / \mathfrak{G}_{k+1}(P)_{0}$, and show that under some assumptions, this space is a finite-dimensional smooth manifold. For $A \in \mathfrak{A}_{k}(P)_{0}$, let us put:

$$
\begin{gathered}
C G_{A}^{k}=\left\{A^{\prime} \in \mathfrak{A}_{k}(P)_{0} \mid\left(d_{A}\right)_{w}^{*}\left(A^{\prime}-A\right)=0\right\} . \\
I: C G_{A}^{k} \times \mathfrak{G}_{k+1}(P)_{0} \rightarrow \mathfrak{A}_{k}(P)_{0} ; \quad(A, g) \mapsto g^{*} A .
\end{gathered}
$$

Let us calculate $d I$ at $(A$, id $)$. If $u \in W_{w}^{k+1}(Y ; \operatorname{Ad}(P))$ and $v \in W_{w}^{k}(Y ; \operatorname{Ad}(P) \otimes$ $\Lambda^{1}$ ), one has:

$$
\left.\frac{d}{d t}(\exp t u)^{*}(A+t v)\right|_{t=0}=d_{A} u+v .
$$

Thus $d I$ is given by:

$$
\begin{gathered}
d I=1 \oplus d_{A}: \operatorname{ker}\left(d_{A}\right)_{w}^{*} \cap W_{w}^{k}\left(Y ; \operatorname{Ad} P \otimes T^{*} Y\right) \times W_{w}^{k+1}(Y ; \operatorname{Ad} P) \\
\rightarrow W_{w}^{k}\left(Y ; \operatorname{Ad} P \otimes T^{*} Y\right) .
\end{gathered}
$$

Since $d_{A}$ has closed range, it is clear that the above map is an isomorphism at every $(A, \mathrm{id})$.

Corollary 1.2 There are neighborhoods $U \subset \mathfrak{A}_{k}(P)_{0}$ of $A$ and $V \subset \mathfrak{G}_{k+1}(P)_{0}$ of id such that:

$$
I: U \cap C G_{A}^{k} \times V \rightarrow \mathfrak{A}_{k}(P)_{0}
$$

is a homeomorphism into its image. By restriction,

$$
I: U \cap C G_{A}^{k}(P) \cap \widehat{\mathfrak{M}}_{k}(P)_{0} \times V \rightarrow \widehat{\mathfrak{M}}_{k}(P)_{0}
$$

is also a homeomorphism into $\widehat{\mathfrak{M}}_{k}(P)_{0}$.

Now one has the following: 
Proposition 1.2 Suppose $d_{A}^{+}: W_{w}^{k}\left(Y ; \operatorname{Ad} P \otimes T^{*} Y\right) \rightarrow W_{w}^{k-1}\left(Y ; \operatorname{Ad} P \otimes \Lambda_{+}^{2}\right)$ is a surjection for $A \in \widehat{\mathfrak{M}}_{k}(P)_{0}$. Then $\mathfrak{M}_{k}(P)=\widehat{\mathfrak{M}}_{k}(P)_{0} / \mathfrak{G}_{k+1}(P)_{0}$ is a finitedimensional smooth manifold at $[A]$. Its tangent space is naturally isomorphic to $\operatorname{ker} d_{A}^{+} / \operatorname{im} d_{A}$.

Proof This follows from the general facts on group actions [14, page 48]. From the above corollary, it is enough to verify that the above $I$ gives a slice for the projection:

$$
\widehat{\mathfrak{M}}_{k}(P)_{0} \rightarrow \mathfrak{M}_{k}(P)=\widehat{\mathfrak{M}}_{k}(P)_{0} / \mathfrak{G}_{k+1}(P)_{0} .
$$

These can be verified by bootstrapping as [14]. We omit to write it.

\section{C Perturbation of Riemannian metrics}

In order to construct smooth moduli spaces, one uses K Uhlenbeck's generic metric theorem. Let $(g, w)$ be an admissible pair for $Y$. Let us choose a smooth map $h: Y \rightarrow[0, \infty)$ with $h(x) \geq \frac{w}{2}(x)$. Then one introduces the following Banach manifold:

$$
\mathfrak{C}=\left\{\phi \in C^{l}(G l(T M)): \lim \sup _{K}\left(\sum_{j=0}^{l} e^{h}\left|\nabla^{j}\left(\phi^{*} g-g\right)\right| K=0, K \text { compact }\right\} .\right.
$$

Let us take $\phi \in \mathfrak{C}$, and put $g^{\prime}=\phi^{*} g$. Then one has the AHS complex with respect to $g^{\prime}$ :

$$
0 \longrightarrow W_{w}^{k+1}\left(\left(Y, g^{\prime}\right) ; \Lambda^{0}\right) \stackrel{d}{\longrightarrow} W_{w}^{k}\left(\left(Y, g^{\prime}\right) ; \Lambda^{1}\right) \stackrel{d^{+}}{\longrightarrow} W_{w}^{k-1}\left(\left(Y, g^{\prime}\right) ; \Lambda_{+}^{2}\right) \longrightarrow 0 .
$$

One sees that this is a Fredholm complex with the same index as the unperturbed one. Our aim in this section is to show the following:

Proposition 1.3 Suppose $Y$ is indefinite. Then by a small perturbation of the Riemannian metric, one has no orbit of reducible connections in $\mathfrak{M}_{k}(P)$. In particular $\mathfrak{M}_{k}(P)$ is a smooth manifold.

In order to verify this, we follow [14. Let us take any $L^{2}$ ASD connection $A_{0}$ over $Y$, and consider $\mathfrak{A}_{k}(P)_{0}=\left\{A_{0}+a \mid a \in W_{w}^{k}\left(Y ; \operatorname{Ad}(P) \otimes \Lambda^{1}\right)\right.$. Then one introduces a $\mathfrak{G}_{k+1}(P)_{0}$ equivariant map:

$$
P_{+}: \mathfrak{A}_{k}(P)_{0} \times \mathfrak{C} \rightarrow W_{w}^{k-1}\left((Y, g) ; \Lambda_{+}^{2}\right)
$$

by $P_{+}(A, \phi)=P_{+}(g)\left(\phi^{-1}\left(F_{A}\right)\right)$, where $P_{+}(g)$ is the projection to the self dual part with respect to $g$. Notice that this map is well-defined since $A_{0}$ is $L^{2}$ ASD with respect to $g$. Let us put $\overline{\mathfrak{M}}_{k}(P)=P_{+}^{-1}(0)$. 
Proposition 1.4 [14] $\overline{\mathfrak{M}}_{k}(P) \cap \mathfrak{A}_{k}^{*}(P) \times \mathfrak{C} / \mathfrak{G}_{k+1}(P)_{0}$ is a smooth Banach manifold.

Proof We sketch its proof. First we see that $d P_{+}$is surjective at any $(A, \varphi)$ with $P_{+}(A, \varphi)=0$. Then it follows that $\overline{\mathfrak{M}}_{k}(P)$ is a Banach manifold on which $\mathfrak{G}_{k+1}(P)_{0}$ acts. Then as before by making a slice for the action, one gets the result.

Let $\mathfrak{c}$ be the Lie algebra of $\mathfrak{C}$. Then $d P_{+}$splits as:

$$
d P_{+}=d_{1} P_{+} \oplus d_{2} P_{+}: W_{w}^{k}\left(Y ; \operatorname{Ad}(P) \otimes \Lambda^{1}\right) \oplus \mathfrak{c} \rightarrow W_{w}^{k-1}\left(Y ; \operatorname{Ad}(P) \otimes \Lambda_{+}^{2}\right),
$$

where $d_{1} P_{+}(\alpha)=P_{+}(g)\left(\varphi^{-1}\left(d_{A}(\alpha)\right)\right) \mid(u, \varphi)$, and $d_{2} P_{+}(r)=P_{+}\left(\left(\varphi^{-1}\right)^{*}\left(r^{*} F\right)\right)$. We show that the differential of $P_{+}$is surjective - notice that

$$
\operatorname{ker} d_{A} \cap W_{w}^{k+1}(Y ; \operatorname{Ad}(P))=0 .
$$

Let us consider the AHS complex:

$$
0 \longrightarrow W_{w}^{k+1}(\operatorname{Ad}(P)) \stackrel{d_{A}}{\longrightarrow} W_{w}^{k}\left(\operatorname{Ad}(P) \otimes \Lambda^{1}\right) \stackrel{d_{A}^{+}}{\longrightarrow} W_{w}^{k-1}\left(\operatorname{Ad}(P) \otimes \Lambda_{+}^{2}\right) \longrightarrow 0 .
$$

Since this is Fredholm, one sees $d P_{+}$has finite codimension. Let us take a representative $u \in$ coker $d P_{+}$with $\left(d_{A}^{+}\right)^{*}(u)=0$. Then one has $\left(d_{A}^{+}\right)^{*}\left(e^{w} u\right)=0$. Then one has the equations, $d_{A}\left(F_{A}\right)=d_{A}^{*}\left(F_{A}\right)=d_{A}(v)=d_{A}^{*}(v)=0$, where $v=\varphi^{*}\left(e^{w} u\right)$. Then the same argument as [14, page 56] shows that on an open dense subset of $Y, F_{A}$ can be expressed as $\alpha \otimes a \in \Lambda_{+}^{2} \otimes \operatorname{Ad}(P)$, with $|a|=1$ (pointwise norm) and $d_{A}(a)=0$. By the irreducibility, it follows $a=0$, which contradicts to non triviality of $p_{1}(P)$. This completes the proof.

Proposition 1.5 Let us fix $A_{0}$, an $L^{2} A S D$ connection with respect to $g$. Then for a Baire set of $\phi \in \mathfrak{C}$, there are no reducible connections in $\mathfrak{A}_{k}(P)_{0}$ with respect to $\phi^{*}(g)$.

Proof Suppose $A_{0}$ is reducible and denote the corresponding $U(1)$-connection by the same $A_{0}$. Let $P$ be a $G=U(1)$-bundle. Then, similarly to before, one puts:

$$
\begin{aligned}
& \mathfrak{A}_{k}(P)_{0}=\left\{A_{0}+a \mid a \in W_{w}^{k}\left(Y ; \Lambda^{1}\right)\right\}, \\
& \mathfrak{G}_{k+1}(P)_{0}=\left\{h \in W_{l o c}^{k+1}(Y ; \operatorname{Aut}(E)) \mid h-\mathrm{id} \in W_{w}^{k+1}\left(Y ; T^{*} Y \otimes \mathbb{C}\right)\right\} .
\end{aligned}
$$

Then $\mathfrak{G}_{k+1}(P)_{0}$ acts on $\mathfrak{A}_{k}(P)_{0}$. Now, as above, one considers $P_{+}: \mathfrak{A}_{k}(P)_{0} \times$ $\mathfrak{C} \rightarrow W_{w}^{k-1}\left(\left(Y, g^{\prime}\right) ; \Lambda_{+}^{2}\right)$. As [14, if $d P_{+}$is not surjective, then it follows $F_{A_{0}}=$ 0 . This shows that $d P_{+}$is surjective. 
Now one has a smooth Banach manifold $\overline{\mathfrak{M}}_{k}(P)=P_{+}^{-1}(0) / \mathfrak{G}_{k+1}(P)_{0}$. Let us consider a Fredholm map between Banach manifolds $\bar{\pi}: \overline{\mathfrak{M}}_{k}(P) \rightarrow \mathfrak{C}$. Its Fredholm index is the one of the following:

$$
0 \longrightarrow W_{w}^{k+1}\left(Y ; \Lambda^{0}\right) \stackrel{d}{\longrightarrow} W_{w}^{k}\left(Y ; \Lambda^{1}\right) \stackrel{d^{+}}{\longrightarrow} W_{w}^{k-1}\left(Y ; \Lambda_{+}^{2}\right) \longrightarrow 0 .
$$

One has $H^{0}(\mathrm{AHS})=H^{1}(\mathrm{AHS})=0$, and $H^{2}(\mathrm{AHS}) \geq b_{+}^{2}$. This is also the case when one perturbs the Riemannian metrics slightly. In particular, if $b_{+}^{2}>0$, then one has $\operatorname{dim} H^{1}-\operatorname{dim} H^{2}<0$. Then the Sard-Smale theorem shows that for a Baire set of $\mathfrak{C}$, there are no $L^{2}$ ASD connections over nontrivial line bundles. This shows that there are no orbits of reducible ASD connections in $\mathfrak{M}_{k}(P)$, for a Baire set of $\mathfrak{C}$. This completes the proof.

Let us put the projection $\pi: \overline{\mathfrak{M}}_{k}(P) \rightarrow \mathfrak{C}$. Then the direct application of [14] to this case shows:

Corollary 1.3 14] Suppose $b_{+}^{2}>0$. Then a Baire set of $\phi \in \mathfrak{C}$ exists such that $\overline{\mathfrak{M}}_{k}(\phi) \equiv \pi^{-1}(\phi)$ are smooth finite dimensional manifolds.

\section{A complete Riemannian metric on int $W_{0}$}

Roughly speaking the set of the anti-self-dual connections modulo equivalence forms a finite-dimensional manifold, in the case when the base four-manifold is closed. Then dimension is equal to the Fredholm index of the elliptic complex, the Atiyah-Hitchin-Singer complex. One may make a Fredholm operator for a four-manifold with boundary, using suitable complete Riemannian metrics on its interior. In this case one uses the weighted Sobolev spaces on the elliptic operator. For our purpose this is a basic construction, and later we will use infinitely many such open Riemannian manifolds. In fact by gluing out all of them iteratively, we will get another complete Riemannian manifold such that the Atiyah-Hitchin-Singer complex becomes Fredholm between the weighted Sobolev spaces. The aim in later sections is to construct admissible pairs over Casson handles of bounded type.

\section{A A specified embedding of $W_{0}$ into $\mathbb{R}^{N}$}

Let $W_{0}$ be a compact manifold with boundary of arbitrary dimension $n$. Let us choose compact submanifolds with boundary $M_{1}, N_{0} \subset \partial W_{0}$ of the same dimension, with empty intersection, $M_{1} \cap N_{0}=\emptyset$. Suppose $M_{1}$ and $N_{0}$ are 
diffeomorphic to each other, and denote $\widetilde{W}_{0}=\operatorname{int} W_{0} \cup M_{1} \cup N_{0}$. Let us take a countable number of $\widetilde{W}_{0}$ and index them by $\widetilde{W}_{0}^{i}=\widetilde{W}_{0}, i \in \mathbf{Z}$. Then one constructs an open manifold $Y$ by $\cup \widetilde{W}_{0}^{i}$ identifying $M_{1}^{i}$ with $N_{0}^{i+1}$ :

$$
Y=\ldots \widetilde{W}_{0}^{i} \cup_{M_{1}^{i} \sim N_{0}^{i+1}} \widetilde{W}_{0}^{i+1} \cup \ldots
$$

There is a natural smooth $\mathbb{Z}$-action on $Y$, and we call $Y$ a periodic open manifold. There are obvious notions of periodic vector bundles over $Y$, and periodic differential operators over $E$.

In order to define (weighted) Sobolev spaces over $Y$ we need, first of all, a complete Riemannian metric on int $W_{0}$ which extends to the one on $Y$. We recall an explicit picture of end-connected sums along $M_{1}$ and $N_{0}$. Let us choose smooth embeddings:

$$
\gamma\left(\gamma^{\prime}\right): M_{1}\left(N_{0}\right) \times[0, \infty) \hookrightarrow \partial W_{0} \times[0, \infty) \subset \operatorname{int} W_{0} .
$$

Then we have the following equality (at this stage it is $C^{\infty}$ sense, by smoothing corners):

$$
\text { int } W_{0} \text { Łint } W_{0}=\operatorname{int} W_{0} \backslash \operatorname{im} \gamma \cup \operatorname{int} W_{0} \backslash \operatorname{im} \gamma^{\prime}
$$

where we identify $\partial M_{1} \times[0, \infty) \cup M_{1} \times\{0\}$ with $\partial N_{0} \times[0, \infty) \cup N_{0} \times\{0\}$. In order to make this construction compatible with the metric on int $W_{0}$, we choose a particular style of Riemannian metric on int $W_{0}$ as follows. Let us choose an embedding:

$$
I: W_{0} \hookrightarrow \mathbb{R}^{N+2}
$$

with the following six properties:

(1) Let us choose a small neighborhood of $\partial W_{0}$ in $W_{0}$ and identify it with $\partial W_{0} \times[0, \epsilon] \equiv W_{0}(\epsilon), \partial W_{0}=\partial W_{0} \times\{0\}$. Then for any $\tau \in[0, \epsilon]$, we have the following:

$$
W_{0} \cap \mathbb{R}^{N+1} \times[0, \epsilon]=W_{0}(\epsilon), \quad W_{0}(\epsilon) \cap \partial W_{0} \times \tau \subset \mathbb{R}^{N+1} \times \tau .
$$

(2) For a smaller $\epsilon^{\prime}<\epsilon, p\left(\left\{W_{0}\left(\epsilon^{\prime}\right) \cap \partial W_{0} \times \tau\right\}\right) \subset \mathbb{R}^{N+1}$ is independent of $\tau \in\left[0, \epsilon^{\prime}\right]$, where $p: \mathbb{R}^{N+2} \rightarrow \mathbb{R}^{N+1}$ is the obvious projection.

(3) $I \mid W_{0} \backslash W_{0}(\epsilon)$ is a $C^{\infty}$ embedding (ie without corners).

(4) Let $\bar{M}_{1}$ and $\bar{N}_{0}$ be tubular neighborhoods of $M_{1}$ and $N_{0}$ in $\partial W_{0}$ respectively. As before we identify small neighborhoods of $\partial \bar{M}_{1}$ and $\partial \bar{N}_{0}$ with $\partial M_{1} \times(0, \delta] \equiv M_{1}(\delta)$ and $\partial N_{0} \times(0, \delta] \equiv N_{0}(\delta)$ respectively. Then we have the following:

(a) $\bar{M}_{1}$ and $\bar{N}_{0}$ are diffeomorphic to $M_{1}$ and $N_{0}$ respectively, 
(b) $\partial W_{0} \cap \mathbb{R}^{N} \times\{0\}=M_{1}, \quad \partial W_{0} \cap \mathbb{R}^{N} \times\{1\}=N_{0}$,

(c) for $0<\tau<\delta, M_{1}(\delta) \cap \mathbb{R}^{N} \times \tau$ and $N_{0}(\delta) \cap \mathbb{R}^{N} \times\{1-\tau\}$ are diffeomorphic to $\partial M_{1}$ and $\partial N_{0}$ respectively.

(5) $W_{0}(\epsilon) \cap \mathbb{R}^{N} \times \tau \times[0, \epsilon]$ and $W_{0}(\epsilon) \cap \mathbb{R}^{N} \times\{1-\tau\} \times[0, \epsilon], \tau \in(0, \delta]$, are diffeomorphic to $\partial M_{1} \times[0, \epsilon] \cup M_{1}$ and $\partial N_{0} \times[0, \epsilon] \cup N_{0}$ respectively.

Moreover $W_{0}(\epsilon) \cap \mathbb{R}^{N} \times \tau \times[0, \epsilon] \subset \mathbb{R}^{N} \times\{0\} \times[0, \epsilon]$ and $W_{0}(\epsilon) \cap \mathbb{R}^{N} \times\{1-$ $\tau\} \times[0, \epsilon] \subset \mathbb{R}^{N} \times\{0\} \times[0, \epsilon]$ are independent of $\tau$.

(6) Let us denote $\bar{M}_{1}(\tau)=W_{0}(\epsilon) \cap \mathbb{R}^{N} \times\{\tau\} \times[0, \epsilon]=\left\{(a, b, c) \in \bar{M}_{1}(\delta) \cap\right.$ $\left.\mathbb{R}^{N} \times \mathbb{R} \times \mathbb{R}\right\}$. We have similar notations for $N_{0}$. Then we have $\bar{N}_{0}(\tau)=$ $\bar{M}_{1}^{\sharp}(\tau) \equiv\left\{(a, 1-b, c) \mid(a, b, c) \in \bar{M}_{1}(\tau)\right\}, \tau \in(0, \delta]$.

Let us denote $\widetilde{W_{0}^{\prime}}=W_{0} \backslash W_{0} \cap \mathbb{R}^{N} \times\{0,1\} \times[0, \epsilon]$. Then $I \mid \widetilde{W}_{0}^{\prime}$ is a smooth embedding with corners. Let us denote by $g_{0}$ the Riemannian metric on $\widetilde{W}_{0}^{\prime}$ induced from the standard one on $\mathbb{R}^{N+2}$ through $I$. Notice important properties of $g_{0}$; for $\epsilon^{\prime}<\epsilon$ :

$$
g_{0}\left|W_{0}\left(\epsilon^{\prime}\right)=\left(g_{0} \mid \partial W_{0}\right) \times d y^{2}, \quad g_{0}\right| \bar{M}_{1}((0, \delta]) \cap W_{0}\left(\epsilon^{\prime}\right)=\left(g_{0} \mid \partial M_{1}\right) \times d x^{2} \times d y^{2}
$$

where we have the coordinates $(t, x, y) \in \mathbb{R}^{N} \times \mathbb{R} \times \mathbb{R}$. We have a similar expression for $g_{0} \mid \bar{N}_{0}(\delta)$.

Now we define the desired Riemannian metric on int $W_{0}$ as follows. Let us put:

$$
\begin{aligned}
\widetilde{W}_{0}=\widetilde{W}_{0}^{\prime} \cup\left[\partial W_{0} \backslash\left\{M_{1} \cup N_{0}\right\}\right] \times(-\infty, 0] \cup & \\
{\left[\partial M_{1} \times(-\infty, \epsilon] \cup M_{1} \times\{\epsilon\}\right] \times(-\infty, 0] \cup } & \\
& {\left[\partial N_{0} \times(-\infty, \epsilon] \cup N_{0} \times\{\epsilon\}\right] \times[1, \infty) . }
\end{aligned}
$$

Clearly $\widetilde{W}_{0}$ is diffeomorphic to int $W_{0}$. On the other hand, by the above remark, there is a natural extension of $g_{0}$ to $g \mid \widetilde{W}_{0}$. This is the desired complete Riemannian metric. Later we will use the following notations. Let us choose large $t \gg 0$ and $s \gg 0$. Then we denote:

$$
\begin{aligned}
& \widetilde{W}_{0}(t)=\left[\partial W_{0} \backslash\left\{M_{1} \cup N_{0}\right\}\right] \times\{-t\} \\
& \cup \partial M_{1} \times\{-t\} \times(-\infty, 0] \\
& \cup \cup N_{0} \times\{-t\} \times[1, \infty), \\
& M_{1}(s)=\left\{\partial M_{1} \times(-\infty, \epsilon] \cup M_{1} \times\{\epsilon\}\right\} \times\{-s\} .
\end{aligned}
$$

We denote $M_{1}\left(\left[S_{0}, S_{1}\right]\right)=\cup_{s \in\left[S_{0}, S_{1}\right]} M_{1}(s)$. We have a similar notation $N_{0}(s)$. 


\section{B Casson handles as Riemannian manifolds}

Let us generalize the previous construction over the triple $\left(W_{0}, M_{1}, N_{0}\right)$ as follows. Suppose we have $n+1$ submanifolds $M_{0}, M_{1}, \ldots, M_{n}$ in $\partial W_{0}$ such that they are disjoint from each other, $M_{i} \cap M_{j}=\emptyset$. Then by choosing an appropriate embedding $I: W_{0} \hookrightarrow \mathbb{R}^{N}$, one can construct a complete Riemannian metric on int $W_{0}$ as 2.A. Then one expresses this as $\widetilde{W}_{n}$ :

$\widetilde{W}_{n}=\widetilde{W}_{0}^{\prime} \cup\left[\partial W_{0} \backslash \cup_{i} M_{i}\right] \times(-\infty, 0] \cup_{i}\left[\partial M_{i} \times(-\infty, \epsilon] \cup M_{i} \times\{\epsilon\}\right] \times(-\infty, 0]$.

Let $\Sigma$ be a Riemann surface of genus $g \geq 1$, and $\bar{\Sigma}$ be its solid Riemann surface. Thus $\bar{\Sigma}$ is a three-manifold with boundary $\Sigma$. Let us put $W_{g}=\bar{\Sigma} \times[0,1]$, and take $g$ embeddings of $S^{1} \times D^{2}$ into $\partial W_{g}$ which represents a generating set of $\pi_{1}\left(W_{g}\right)$. We denote their images by $M_{1}, \ldots, M_{g}$. One may assume that all $M_{i}$ and $M_{j}$ are disjoint. A kinky handle with $k$ kinks is expressed as $\left(W_{k}, N_{0}, M_{1}, \ldots, M_{k}\right)$, where $N_{0}$ is another embedding of $S^{1} \times D^{2}$ into $\partial W_{k}$. The isotopy type of $N_{0}$ is determined by the sign $( \pm, \cdots \pm$ ) ( $k$ times). There are exactly $k+1$ diffeomorphism types of kinky handles with $k$ kinks, since the diffeomorphism type of the kinky handle is invariant under permutation of the signs.

A Casson handle is constructed by taking end connected sums of kinky handles infinitely many times. It is expressed by an infinite tree (embedded in $\mathbb{R}^{2}$ ) with additional information. Let us introduce the following:

Definition 2.1 Let $T$ be an infinite tree with only one end point $*$. $T$ is said to be a signed tree if every edge $e$ is assigned one of + or - .

Let $n(v)+1$ be an integer which is the number of edges of $T$ with common end-point $v$. A signed tree $T$ is said to be of bounded multiplicity if there exists a constant $C$ with $C \geq n(v)$ for any $v \in T$.

Any tree with one end-point $*$ admits a natural distance from $*$. One will denote it by || . Let $T$ be a signed tree with one end-point $*$. Let us take any vertex $v \in T$. Then there exists a unique edge $e$ on $T$ such that one of the end-point of $e$ is $v$, and the other $v^{\prime}$ satisfies $\left|v^{\prime}\right|<|v|$. To specify this $e$, one denotes it as $e(v)$. Let us denote finite subtrees by $T_{v}=\cup_{j=0}^{n(v)} e_{j}$, where all the edges $e_{j}$ has $v$ as one end-point, and $e_{0}=e(v)$. Recall that each $e_{j}$ is assigned with \pm . Since $e_{j}, 1 \leq j \leq n(v)$, are all assigned one of \pm , the set $\left\{e_{1}, \ldots, e_{n(v)}\right\}$ determines an isotopy class of smooth embedding $N_{0} \cong S^{1} \times D^{2} \hookrightarrow \partial W_{n(v)}$, where $W_{n(v)}$ is diffeomorphic to $\bar{\Sigma}_{n(v)} \times[0,1]$ and $e(v)$ corresponds to $N_{0}$. This class determines a diffeomorphism type of a kinky handle. Thus every 
$T_{v}$ corresponds to data $\left(W_{n(v)}, N_{0}, M_{1}, \ldots, M_{n(v)}\right)$, a kinky handle with $n(v)$ kinks as described above. In particular one may assign a complete Riemannian manifold $\widetilde{W}_{v}$ for every vertex $v \in T$.

Let us take an edge $e$ with end-points $v$ and $v^{\prime}$, and assign a large number $S \gg 0$. Then one can make end-connected sums of $\widetilde{W}_{v}$ with $\widetilde{W}_{v^{\prime}}$ as:

$$
\widetilde{W}_{v^{\prime}} \natural_{e, S} \widetilde{W}_{v} \equiv \widetilde{W}_{v^{\prime}} \backslash M_{e}([S, \infty)) \llbracket \widetilde{W}_{v} \backslash N_{v}([S, \infty)) .
$$

One will also denote the set $(T, S)$ by $T$, and also call it a signed tree. For every $e$, one may connect $\widetilde{W}_{v}$ and $\widetilde{W}_{v^{\prime}}$ as above. By iterating these end-connected sums for every edge, one gets a complete Riemannian four-manifold $C H(T)$. The diffeomorphism type of this space, is the Casson handle corresponding to $T$.

We denote $\left(W_{0}, N_{0}, M_{1}\right)$ as the simplest kinky handle. Then $\tilde{Y}(S)$ is a periodic Riemannian four-space:

$$
\tilde{Y}(S)=\cup_{j \in \mathbb{Z}} \widetilde{W}_{0}^{j}([-S, S]), \quad C H\left(\mathbb{R}_{+}\right)=\widetilde{Y}(S)_{0}=\cup_{j \in \mathbb{N}} \widetilde{W}_{0}^{j}([-S, S])
$$

where $\widetilde{W}_{0}^{j}([-S, S])=\widetilde{W}_{0}^{j} \backslash N_{0}([S, \infty)) \cup M_{1}([S, \infty))$, and one enumerates the same $\widetilde{W}_{0}$ by $\widetilde{W}_{0}^{j}, j \in \mathbb{Z}$. Even though $C H\left(\mathbb{R}_{+}\right)$has very complicated smooth structure, $\widetilde{Y}(S)$ is diffeomorphic to $\mathbb{R}^{4}$, and so it would not appropriate to write it as $C H(\mathbb{R})$.

Let $\left(W_{l}, N_{0}, M_{1}, \ldots, M_{l}\right)$ be a kinky handle with $l$ kinks. Then one defines the following another Riemannian manifolds. Let us choose any $1 \leq j \leq l$, say $j=1$. Now one defines:

$$
\begin{aligned}
& V(S, l)_{0}=\widetilde{W}_{l} \backslash N_{0}([S, \infty)) \cup_{k=1}^{l} M_{k}([S, \infty)), \\
& Y(S, l, 1)_{0}=V(S, l)_{0} \cup_{j=2}^{l} \cup_{M_{j}(S) \sim N_{0}(S)} \widetilde{Y}(S)_{0} \backslash N_{0}([S, \infty)), \\
& Y(S, l, 1)=Y(S, l, 1)_{0} / N_{0}(S) \sim M_{1}(S), \\
& \tilde{Y}(S, l, 1)_{0}=\cup_{j \in \mathbb{N}} \cup_{M_{1}^{j}(S) \sim N_{0}^{j+1}(S)} Y(S, l, 1)_{0}^{j}, \\
& \tilde{Y}(S, l, 1)=\cup_{j \in \mathbb{Z}} \cup_{M_{1}^{j}(S) \sim N_{0}^{j+1}(S)} Y(S, l, 1)_{0}^{j} .
\end{aligned}
$$

There is an infinite signed tree with a base point $\left(T_{l, 1}\right)_{0}$ such that $\tilde{Y}(S, l, 1)_{0}$ is diffeomorphic to $\mathrm{CH}\left(\left(T_{l, 1}\right)_{0}\right)$.

Let us take $l$ half-lines $\mathbb{R}_{+}, \ldots \mathbb{R}_{+}(l$ times $)$ where on each $\mathbb{R}_{+}$the same sign is given. Signs on two different half-lines may be mutually different. Then one identifies all $\mathbb{R}_{+}$at 0 . The result is a connected signed infinite tree with a base point. One denotes it by $T_{0}^{l}$. $\left(T_{l, 1}\right)_{0}$ can be expressed as $\mathbb{R}_{+} \cup_{j \in \mathbb{N}}\left(T_{0}^{l-1}\right)^{j}$, 
where $\left(T_{0}^{l-1}\right)^{j}$ are the same $\left(T_{0}^{l-1}\right)$ indexed by $j \in \mathbb{N}$, and on $\mathbb{R}_{+}$the same sign is given.

Let us define:

$$
\begin{aligned}
& Y(S, l, m, 1)_{0} \equiv V(S, l)_{0} \cup_{j=2}^{l} \cup_{M_{1}^{j}(S) \sim N_{0}^{1}(S)} \tilde{Y}(S, m, 1)_{0}, \\
& Y(S, l, m, 1)=Y(S, l, m, 1)_{0} / N_{0}(S) \sim M_{1}(S), \\
& \widetilde{Y}(S, l, m, 1)_{0}=\cup_{j \in \mathbb{N}} \cup_{M_{1}^{j}(S) \sim N_{0}^{j+1}(S)} Y(S, l, m, 1)_{0}^{j}, \\
& \widetilde{Y}(S, l, m, 1)=\cup_{j \in \mathbb{Z}} \cup_{M_{1}^{j}(S) \sim N_{0}^{j+1}(S)} Y(S, l, m, 1)_{0}^{j} .
\end{aligned}
$$

Similarly one has $\tilde{Y}(S, l, m, 1)_{0}=C H\left(\left(T_{l, m, 1}\right)_{0}\right)$. Let us take signed infinite trees $\left(T_{m, 1}\right)_{0}, \ldots,\left(T_{m, 1}\right)_{0}$ ( $l$ times). Then one identifies all $\left(T_{m, 1}\right)_{0}$ at 0 . The result is a connected signed infinite tree with a base point. One denotes it by $\left(T_{m, 1}\right)_{0}^{l}$. As above, $\left(T_{l, m, 1}\right)_{0}$ can be expressed as $\mathbb{R}_{+} \cup_{j \in \mathbb{N}}\left[\left(T_{m, 1}\right)_{0}^{l-1}\right]^{j}$, where $\left[\left(T_{m, 1}\right)_{0}^{l-1}\right]^{j}$ are the same $\left(T_{m, 1}\right)_{0}^{l-1}$ indexed by $j \in \mathbb{N}$, and on $\mathbb{R}_{+}$the same sign is given. Similarly one uses a notation $\left(T_{l, m, 1}\right)_{0}^{k}$, which one identifies $k$ $\left(T_{l, m, 1}\right)_{0}$ at 0 . Then using this, one can obtain $\left(T_{k, l, m, 1}\right)_{0}$ by a similar method.

By iteration, one obtains $Y(S, l, m, n, 1)$ and $\tilde{Y}(S, l, m, n, 1)_{0}=C H\left(\left(T_{l, m, n, 1}\right)_{0}\right)$ as above. Inductively one can obtain $C H\left(\left(T_{n_{1}, \ldots, n_{k}, 1}\right)_{0}\right)$ by iteration. One calls $\left(T_{n_{1}, \ldots, n_{k}, 1}\right)_{0}$ a homogeneous tree of bounded type.

Let $\bar{n}=\left\{n_{1}, n_{2}, \ldots, n_{l}, \ldots\right\}$ be an infinite set of positive integers. One can iterate the previous construction infinitely many times. Then one gets a complete Riemannian manifold:

$$
C H\left(\left(T_{\bar{n}}\right)_{0}\right)=C H\left(\left(T_{n_{1}, n_{2}, \ldots}\right)_{0}\right)
$$

where we call $\left(T_{\bar{n}}\right)_{0}$ a homogeneous tree. If there is a bound $C$ with $n_{j} \leq C$ for all $j$, then we call it a homogeneous tree of bounded multiplicity.

\section{Some properties of elliptic operators over $\widetilde{W}_{0}$}

\section{A Spectral decomposition}

Throughout this paper, one uses the following lemmas. Let $P$ be an order-1 elliptic differential operator over a complete Riemannian manifold $W$ of bounded geometry. Let $w$ be a weight function over $W$ and weighted Sobolev $k$-norms on $W$ by $|u| W_{w}^{k}=\left(\Sigma_{l \leq k} \int \exp (w)\left|\nabla^{l} u\right|^{2} v o l\right)^{\frac{1}{2}}$. Thus one has a bounded map $P: W_{w}^{k+1}(W) \rightarrow W_{w}^{k}(W)$. Let us denote by $P_{w}^{*}$ the formal adjoint operator with respect to the weighted $L_{w}^{2}$ inner product. 
Lemma 3.1 Suppose $\operatorname{Spec} \Delta=P_{w}^{*} \circ P$ is contained in $\left[\lambda^{2}, \infty\right)$ for some $\lambda>0$. Then $P: W_{w}^{k+1} \rightarrow W_{w}^{k}$ has closed range.

Similarly $P_{w}^{*}: W_{w}^{k+1} \rightarrow W_{w}^{k}$ also has closed range.

Proof Let us consider the first statement. One verifies this by induction. Let $k=0$ and let $\left\{u_{i}\right\}$ be a sequence in $W_{w}^{1}$ with $\left|P\left(u_{i}\right)\right| L_{w}^{2} \rightarrow 0$. Since one has the estimate $|P(u)| L_{w}^{2} \geq \lambda|u| L_{w}^{2}$, one sees $\left|u_{i}\right| L_{w}^{2} \rightarrow 0$. Moreover by the elliptic estimate:

$$
|u| W_{w}^{k+1} \leq C_{k}\left(|P(u)| W_{w}^{k}+|u| W_{w}^{k}\right), \quad k \geq 0
$$

for some constants $C_{k}$, one concludes $\left|u_{i}\right| W_{w}^{1} \rightarrow 0$. This shows the result for $k=0$.

We show by induction that if a sequence $\left\{u_{i}\right\}$ in $W_{w}^{k+1}$ satisfies $\left|P\left(u_{i}\right)\right| W_{w}^{k} \rightarrow 0$, then $\left|u_{i}\right| W_{w}^{k} \rightarrow 0$. Then by the elliptic estimate, one gets the conclusion. Suppose the result holds for $k \leq k_{0}$, and take a sequence $\left\{u_{i}\right\}$ in $W_{w}^{k_{0}+2}$ with $\left|P\left(u_{i}\right)\right| W_{w}^{k_{0}+1} \rightarrow 0$. Then since $\left|P\left(u_{i}\right)\right| W_{w}^{k_{0}}$ also converges to 0 , one knows that $\left|u_{i}\right| W_{w}^{k_{0}}$ converges to 0 by the induction hypothesis. By the elliptic estimate, one gets the result for $k_{0}+1$. This completes the proof of the first statement.

Next let us consider the second statement. Let $H^{\perp} \subset L_{w}^{2}$ be a closed subspace which consists of the orthogonal complement of $H \equiv P\left(W_{w}^{1}\right) \subset L_{w}^{2}$. Notice the following relation:

$$
\Delta\left(W_{w}^{k+2}\right)=P_{w}^{*}\left(H \cap W_{w}^{k+1}\right) \subset W_{w}^{k}
$$

Now let $\left\{v_{i}\right\}$ be a sequence in $H \cap W_{w}^{1}$ with $\left|P_{w}^{*}\left(v_{i}\right)\right| L_{w}^{2} \rightarrow 0$. Since one may express $v_{i}=P\left(u_{i}\right)$ for $u_{i} \in W_{w}^{2}$, one has $\left|\Delta\left(u_{i}\right)\right| L_{w}^{2} \rightarrow 0$. Thus one has $\left|u_{i}\right| L_{w}^{2} \rightarrow 0$. Then by Cauchy-Schwartz, one has the estimate $\left|P\left(u_{i}\right)\right|^{2} L_{w}^{2} \leq$ $\left|\Delta\left(u_{i}\right)\right| L_{w}^{2}\left|u_{i}\right| L_{w}^{2}$. This shows $\left|v_{i}\right| L_{w}^{2} \rightarrow 0$. By the elliptic estimate, one sees $\left|v_{i}\right| W_{w}^{1} \rightarrow 0$. Then by proceeding similarly as above, one verifies the conclusion by induction. This completes the proof.

Let $W$ and $w$ be as above, and consider an elliptic complex:

$$
0 \longrightarrow E_{0} \stackrel{d_{0}}{\longrightarrow} E_{1} \stackrel{d_{1}}{\longrightarrow} E_{2} \longrightarrow 0
$$

over vector bundles on $W$.

Lemma $3.2(1)$ Suppose $d_{0}: W_{w}^{k+1}\left(E_{0}\right) \rightarrow W_{w}^{k}\left(E_{1}\right)$ has closed range, and ker $d_{0}=0$. Then there exists a constant $C$ such that:

$$
\left|\left(d_{0}\right)_{w}^{*} \circ d_{0}(u)\right| W_{w}^{k}\left(E_{0}\right) \geq C|u| W_{w}^{k+2}\left(E_{0}\right)
$$


for any $u$. In particular $\left(d_{0}\right)_{w}^{*} \circ d_{0}$ gives an isomorphism.

(2) Suppose the corresponding bounded complex:

$$
0 \longrightarrow W_{w}^{k+2}\left(E_{0}\right) \stackrel{d_{0}}{\longrightarrow} W_{w}^{k+1}\left(E_{1}\right) \stackrel{d_{1}}{\longrightarrow} W_{w}^{k}\left(E_{2}\right) \longrightarrow 0
$$

satisfies the following; (1) $d_{0}$ and $d_{1}$ have closed range, (2) the first cohomology group ker $d_{1} / \operatorname{im} d_{0}=0$, and (3) $\operatorname{ker} d_{0}=0$.

Then for $P=\left(d_{0}\right)_{w}^{*} \oplus d_{1}$ and $\Delta=P_{w}^{*} \circ P$, one has $\operatorname{Spec} \Delta \subset[\lambda, \infty)$ for some positive constant $\lambda>0$. Moreover $\left(d_{1}\right)_{w}^{*}$ has closed range.

Proof (1) Suppose the contrary, and take a sequence $\left\{u_{i}\right\} \subset W_{w}^{2}$ with $\mid\left(d_{0}\right)_{w}^{*}$ o $d_{0}\left(u_{i}\right) \mid L_{w}^{2} \rightarrow 0$. Then by Cauchy-Schwartz one has a convergent sequence $\left|d_{0}\left(u_{i}\right)\right| L_{w}^{2} \rightarrow 0$. Then by the elliptic estimate, one has $\left|d_{0}\left(u_{i}\right)\right| W_{w}^{1} \rightarrow 0$. By closedness and the open mapping theorem, this shows existence of non-trivial kernel. This is a contradiction. The rest of the proof follows by induction.

Part (2) follows from (1) and lemma 3.1. This completes the proof.

Let us use the above notations. Let us put $H=L_{w}^{2}\left(E_{1}\right) \cap\left(\operatorname{ker} d_{1}\right)^{\perp}$. Let us consider restriction of $\Delta=\left(d_{1}\right)_{w}^{*} \circ d_{1} \mid H$.

Corollary 3.1 Suppose $d_{0}$ and $d_{1}$ has closed range as above. Then Spec $\Delta \mid H$ is contained in $[\lambda, \infty)$ for some positive $\lambda>0$.

\section{B Analysis over $\widetilde{W}_{0}$}

Let us take the complete Riemannian manifold $\widetilde{W}_{0}$ in 2.A. Let us take a smooth function $w: \widetilde{W}_{0} \rightarrow[0, \infty)$. Then as before one has weighted Sobolev $k$ norms on $\widetilde{W}_{0}$ by $|u| W_{w}^{k}=\left(\Sigma_{l \leq k} \int \exp (w)\left|\nabla^{l} u\right|^{2} v o l\right)^{\frac{1}{2}}$. Then we have the AtiyahHitchin-Singer complex (AHS complex) as:

$$
0 \longrightarrow W_{w}^{k+2}\left(\widetilde{W}_{0} ; \Lambda^{0}\right) \stackrel{d}{\longrightarrow} W_{w}^{k+1}\left(\widetilde{W}_{0} ; \Lambda^{1}\right) \stackrel{d_{+}}{\longrightarrow} W_{w}^{k}\left(\widetilde{W}_{0} ; \Lambda_{+}^{2}\right) \longrightarrow 0
$$

where $d^{+}=(1+*) \circ d$.

Let $M$ be a complete Riemannian 3 manifold without boundary, but possibly be non-compact. We have in mind $M=\widetilde{W}_{0}(t), N_{0}(s)$ or $M_{1}(s)$. Let $g$ be a Riemannian metric on $M$, and denote also by $g$ the product metric on $M \times \mathbb{R}$. Let $\Lambda^{1}(M \times \mathbb{R})$ and $\Lambda_{+}^{2}(M \times \mathbb{R})$ be the exterior differentials on 1 forms and self-dual 2-forms with respect to $g$. Then we have the natural identification:

$$
\Lambda^{1}(M \times \mathbb{R})=p^{*}\left(\Lambda^{1}(M)\right) \oplus p^{*}\left(\Lambda^{0}(M)\right),
$$




$$
\Lambda_{+}^{2}(M \times \mathbb{R})=p^{*}\left(\Lambda^{1}(M)\right)
$$

where $p: M \times \mathbb{R} \rightarrow M$ is the projection. The isomorphisms are given by:

$$
u+v d t \leftrightarrow(u, v), \quad *_{M} u+u \wedge d t \leftrightarrow u .
$$

Let us put $X=M \times \mathbb{R}$. Using the $L^{2}$ adjoint operator (here we do not use any weight functions), we get the elliptic operator $P=d^{*} \oplus d^{+}: \Lambda^{1}(X) \rightarrow$ $\Lambda^{0}(X) \oplus \Lambda_{+}^{2}(X)$. Using the above identification, this is expressed as:

$$
P=d^{*} \oplus d^{+}: p^{*}\left(\Lambda^{0}(M) \oplus \Lambda^{1}(M)\right) \rightarrow p^{*}\left(\Lambda^{0}(M) \oplus \Lambda^{1}(M)\right) .
$$

Let us use $t$ as the coordinate on $\mathbb{R}$. Then by a straightforward calculation, one has the following expression:

$$
P=-\frac{d}{d t}+\left(\begin{array}{cc}
*_{M} d & d \\
d^{*} & 0
\end{array}\right) \equiv-\frac{d}{d t}+Q .
$$

Notice that $Q$ is an elliptic self adjoint differential operator on $L^{2}\left(M ; \Lambda^{0}(M) \oplus\right.$ $\left.\Lambda^{1}(M)\right)$. Notice that if $M$ is closed, then $Q$ is not invertible (one has constant functions). When $M$ is non compact, 0 may be contained in the spectrum of $Q$, even if there are no kernels for $Q$.

Let us introduce a weight function $\tau$ on $X$ as follows. Let $M$ be possibly non-compact as above. Let us choose a positive number $\delta>0$ which will be specified later, and choose a smooth proper function $\tau^{\prime}: \mathbb{R} \rightarrow[0, \infty)$ with $\tau^{\prime}|\mathbb{R} \backslash[-1,1](t)=\delta| t \mid$. Then we define $\tau: M \times \mathbb{R} \rightarrow[0, \infty)$ by $\tau(m, t) \equiv \tau^{\prime}(t)$. Let us introduce weighted Sobolev $k$ norms on $X$ by:

$$
|u| W_{\tau}^{k}=\left(\Sigma_{l \leq k} \int \exp (\tau)\left|\nabla^{l} u\right|^{2} v o l\right)^{\frac{1}{2}} .
$$

We denote these Sobolev weighted spaces by $W_{\tau}^{k}$ or $L_{\tau}^{2}($ for $k=0)$. Then one defines an isometry:

$$
I: L^{2}\left(X, p^{*}\left(\Lambda^{0} \oplus \Lambda^{1}\right)\right) \rightarrow L_{\tau}^{2}\left(X, p^{*}\left(\Lambda^{0} \oplus \Lambda^{1}\right)\right)
$$

by $I(u)=\exp \left(-\frac{\tau}{2}\right) u$. Then one has the equality:

$$
I^{-1} P I=P+\frac{1}{2} \frac{d \tau}{d t}: W^{k+1}\left(X ; p^{*}\left(\Lambda^{0} \oplus \Lambda^{1}\right)\right) \rightarrow W^{k}\left(X ; p^{*}\left(\Lambda^{0} \oplus \Lambda^{1}\right)\right) .
$$

Let $d_{\tau}^{*}$ be the $L_{\tau}^{2}$ adjoint operator, i.e. $\langle u, d(v)\rangle\left|L_{\tau}^{2}=\left\langle d_{\tau}^{*}(u), v\right\rangle\right| L_{\tau}^{2}$, and put $P_{\tau}=d_{\tau}^{*} \oplus d^{+}$. Then we have the following expression:

$$
I^{-1} P_{\tau} I=-\frac{d}{d t}+\left(\begin{array}{cc}
*_{M} d & d \\
d^{*} & -\frac{d \tau}{d t}
\end{array}\right)+\frac{1}{2} \frac{d \tau}{d t} \equiv-\frac{d}{d t}+Q_{\tau} .
$$


We recall the definition of $\widetilde{W}_{0}(t)$ or $M_{1}(s), N_{0}(s)$ in the last paragraph in 2.A. Practically we will apply the next lemma for $M=\widetilde{W}_{0}(t)$. Let us consider the operator:

$$
Q_{\delta}=\left(\begin{array}{cc}
{ }_{M}{ }_{M}+\frac{\delta}{2} & d \\
d^{*} & -\frac{\delta}{2}
\end{array}\right): W^{k+1}\left(M, \Lambda^{1} \oplus \Lambda^{0}\right) \rightarrow W^{k}\left(M, \Lambda^{1} \oplus \Lambda^{0}\right) .
$$

Notice that $Q_{\delta}$ coincides with $Q_{\tau}$ above for $t \geq 1$.

Lemma 3.3 Suppose $M$ is a closed Riemannian manifold. Then for a small $\delta>0$ and $k \geq 0, Q_{\delta}$ gives an isomorphism.

Proof Let us take $(u, v) \in W^{k+1}\left(M, \Lambda^{1} \oplus \Lambda^{0}\right)$, and put $Q_{\delta}(u, v)=(x, y)$. Then we have:

$$
\left(* d+\frac{\delta}{2}\right) u+d v=x, \quad d^{*} u-\frac{\delta}{2} v=y .
$$

For convenience, we recall $*^{2} u=(-1)^{p(n-p)} u, d^{*} u=(-1)^{n p+n+1} * d * u$ for a $p$-form $u$ on an $n$-dimensional manifold. Then from the above, we have:

$$
d^{*} * d u+\frac{\delta}{2} d^{*} u+d^{*} d v=d^{*} x=\left(\left(\frac{\delta}{2}\right)^{2}+d^{*} d\right) v+\frac{\delta}{2} y .
$$

Thus we can solve $v$ from $(x, y)$ as follows:

$$
v=\left(\left(\frac{\delta}{2}\right)^{2}+d^{*} d\right)^{-1}\left(d^{*} x+\frac{\delta}{2} y\right) .
$$

Next we have the following equality:

$$
\begin{gathered}
*^{2} d u+\frac{\delta}{2} * u+* d v=* x=d u+\frac{\delta}{2} * u+* d v . \\
d^{*} d u+\frac{\delta}{2} d^{*} * u+d^{*} * d v=d^{*} * x \\
=d^{*} d u+\frac{\delta}{2} * d u=d^{*} d u+\frac{\delta}{2}\left(x-\frac{\delta}{2} u-d v\right) .
\end{gathered}
$$

Then from this, we have the following equation:

$$
\begin{aligned}
\left(d^{*} d-\left(\frac{\delta}{2}\right)^{2}\right) u & =-\frac{\delta}{2}(x-d v)+d^{*} * x, \\
d d^{*} u & =\frac{\delta}{2} d v+d y .
\end{aligned}
$$


Thus one can again solve $u$ as:

$$
u=\left(\Delta-\left(\frac{\delta}{2}\right)^{2}\right)^{-1}\left(d y+d^{*} * x-\frac{\delta}{2} x+\delta d v\right)
$$

It follows that there is a constant $C=C(M)$ such that Spec $\left|Q_{\delta}\right| \geq C\left(\frac{\delta}{2}\right)^{4}$. Notice the following inequalities:

$$
\begin{aligned}
|(u, v)| W^{k+1} & \leq C\left(\frac{\delta}{2}\right)^{-2}\left|\left(\Delta \pm\left(\frac{\delta}{2}\right)^{2}\right)(u, v)\right| W^{k-1} \\
& \leq C\left(\frac{\delta}{2}\right)^{-4}\left(|x| W^{k}+|y| W^{k}\right) \leq C\left(\frac{\delta}{2}\right)^{-4}\left|Q_{\delta}(u, v)\right| W^{k} .
\end{aligned}
$$

In fact one may assume that $C$ only depends on $\sup _{0 \leq l \leq 2} \sup _{x \in M}\left|\nabla^{l} R\right|(x)$, where $R$ is the curvature form with respect to the Levi-Civita connection. This completes the proof.

Let $\left(P_{\tau}\right)_{\tau}^{*}$ be the formal adjoint of the Sobolev weighted spaces. Then one considers the same analysis for $\left(P_{\tau}\right)_{\tau}^{*}$. Notice the equality:

$$
\left(I^{-1} \circ P_{\tau} \circ I\right)^{*}=I^{-1} \circ\left(P_{\tau}\right)_{\tau}^{*} \circ I .
$$

Thus one can analyze an operator of the form $P_{\tau}^{*}=\frac{d}{d t}+Q_{\delta}$, since $Q_{\delta}$ is self-adjoint over $M$.

Let us take a smooth (non-proper) function:

$$
\tau: \widetilde{W}_{0} \rightarrow[0, \infty)
$$

which is horizontally constant, namely for some positive constant $\delta>0$ we have $\tau \mid \widetilde{W}_{0}(t)=\delta t$ for $t \geq 0$. Using $\tau$, one defines weighted Sobolev spaces $W_{\tau}^{k}\left(\left(\widetilde{W}_{0}, g\right), \Lambda_{*}\right)$. One has the expression $P_{\tau} \mid M_{1}([0, \infty))=-\frac{d}{d s}+Q_{\tau^{\prime}}$ where:

$$
Q_{\tau^{\prime}}=\left(\begin{array}{cc}
* d & d \\
d_{\tau^{\prime}}^{*} & 0
\end{array}\right): W_{\tau^{\prime}}^{k+1}\left(M_{1}(s) ; \Lambda^{1} \oplus \Lambda^{0}\right) \rightarrow W_{\tau^{\prime}}^{k}\left(M_{1}(s) ; \Lambda^{1} \oplus \Lambda^{0}\right)
$$

is an elliptic operator over $M_{1}(s)$, and $\tau^{\prime}=\tau \mid M_{1}(s)$ is its restriction. It follows that $Q_{\tau^{\prime}}$ has closed range with empty kernel. We do not use this fact later. In this case one can not see whether $P_{\tau}$ defined above is Fredholm or not, since one has no simple statement as above for the weighted adjoint operator $\left(Q_{\tau^{\prime}}\right)_{\tau^{\prime}}^{*} \mid W_{\tau^{\prime}}^{k}\left(M_{1}(s) ; \Lambda^{1}\right)$.

Let us take another weight function $\mu: \widetilde{W}_{0} \rightarrow[0, \infty)$, with $\mu \mid M_{1}(s), N_{0}(s) \equiv$ $\delta^{\prime} s$, where we choose a sufficiently small $0<\delta^{\prime} \ll \delta$. Let us put:

$$
w=\tau+\mu: \widetilde{W}_{0} \rightarrow[0, \infty) .
$$


We call $w$ a weight function with weight $\left(\delta, \delta^{\prime}\right)$. Let us use $w$ as the weight function. Then one obtains a bounded operator:

$$
P_{w}=d_{w}^{*} \oplus d^{+}: W_{w}^{k+1}\left(\widetilde{W}_{0} ; \Lambda^{1}\right) \rightarrow W_{w}^{k}\left(\widetilde{W}_{0} ; \Lambda^{0} \oplus \Lambda_{+}^{2}\right) .
$$

Passing through the isometry $I: L^{2} \rightarrow L_{w}^{2}, I(u)=\exp \left(-\frac{w}{2}\right) u, P_{w}$ has the following expression on $M_{1}([0, \infty))$ and $N_{0}([0, \infty))$ :

$$
I^{-1} \circ P_{w} \circ I=-\frac{\partial}{\partial s}+\left(\begin{array}{cc}
* d & d \\
d_{\tau^{\prime}}^{*} & 0
\end{array}\right)+\frac{\delta^{\prime}}{2}\left(\begin{array}{cc}
1 & 0 \\
0 & -1
\end{array}\right) \equiv-\frac{\partial}{\partial s}+Q_{w}^{v} .
$$

On the other hand one has another expression on $\widetilde{W}_{0}([0, \infty))$ :

$$
I^{-1} \circ P_{w} \circ I=-\frac{\partial}{\partial t}+\left(\begin{array}{cc}
* d+\frac{\delta}{2} & d \\
d_{\mu}^{*} & -\frac{\delta}{2}
\end{array}\right)=-\frac{\partial}{\partial t}+Q_{w}^{h} .
$$

\section{C Decay estimate}

Let $M$ be a (possibly non-compact) $n$-dimensional Riemannian manifold of bounded geometry. In practice $M$ stands for $\widetilde{W}_{0}(t)$ (or $N_{0}(s), M_{1}(s)$ ). Let us take a smooth function $\mu: M \rightarrow[0, \infty)$. Let $E$ and $F$ be vector bundles over $M$, and take an elliptic operator:

$$
Q: W_{\mu}^{k+1}(M ; E) \rightarrow W_{\mu}^{k}(M ; F) .
$$

Let us choose $\tau: M \times \mathbb{R} \rightarrow \mathbb{R}_{+}$by $\tau(m, t)=\delta|t|$ for $|t| \geq 1$, and put $w=\tau+\mu$. Then one considers the following operator:

$$
P=-\frac{d}{d t}+Q: W_{w}^{k+1}\left(M \times \mathbb{R}, p^{*} E\right) \rightarrow W_{w}^{k}\left(M \times \mathbb{R} ; p^{*} F\right) .
$$

Let $P_{w}^{*}$ be the formal adjoint operator with respect to the weighted inner product. Then $P_{w}^{*} P$ admits a spectral decomposition. For $u \in W_{w}^{k}\left(M \times \mathbb{R}, p^{*}(E)\right)$, we denote $\operatorname{Spec} u \in[0, \epsilon]$ if $u$ lies in the image of the spectral projection $\mathfrak{P}([0, \epsilon])$.

Suppose the above $Q$ is a Fredholm operator, and is self adjoint with respect to the weighted $L_{\mu}^{2}$ inner product.

Proposition 3.1 24, 13, 28, For sufficiently small $\delta>0$, the above $P$ is Fredholm. Moreover if $Q$ is invertible, then one may choose $\delta=0$. In particular $P$ is also invertible.

Suppose $Q: W_{\mu}^{k+1}(M ; E) \rightarrow W_{\mu}^{k}(M ; F)$ has closed range with $\operatorname{ker} Q=0$. Let us put $\Delta=Q_{\mu}^{*} \circ Q$, and choose a positive constant $\lambda_{0}>0$ with $\operatorname{Spec} \Delta \in$ 
$\left[\lambda_{0}^{2}, \infty\right)$. Then under the above condition, one has the following; for any small $\epsilon \ll \lambda_{0}$, there exist positive constants $C, \delta_{0}>0$ such that for any $u \in W^{k+l}\left(M \times \mathbb{R}, p^{*} E\right)$ with $\operatorname{Spec} u \in[0, \epsilon]$ for $P_{w}^{*} \circ P$, we have the following decay estimate:

$$
|u(, t)| L_{\mu}^{2}(M) \leq C \exp \left(-\delta_{0}|t|\right)|u| L_{\mu}^{2}(M \times[0, \infty)), \quad t \geq 0 .
$$

Proof The first statement follows from the decay estimate. Next let us take $u$ as above, and denote its slice on $M \times s$ by $u_{s}$. Let us put a smooth function $f: \mathbb{R} \rightarrow[0, \infty)$ by $f(s)=\int_{M_{s}} \exp (\mu)\left|u_{s}\right|^{2}$. Then by differentiating, one has the inequalities:

$$
\begin{aligned}
f^{\prime \prime}(s) & =2\left[\int_{M_{s}} \exp (\mu)\left\langle u_{s}^{\prime}, u_{s}^{\prime}\right\rangle+\int_{M_{s}} \exp (\mu)\left\langle u_{s}^{\prime \prime}, u_{s}\right\rangle\right] \\
& \geq 2\left[\int_{M_{s}} \exp (\mu)\left\langle u_{s}^{\prime}, u_{s}^{\prime}\right\rangle+\int_{M_{s}} \exp (\mu)\left\langle(\Delta-\epsilon) u_{s}, u_{s}\right\rangle\right] \\
& \geq 2 \int_{M_{s}} \exp (\mu)\left\langle(\Delta-\epsilon) u_{s}, u_{s}\right\rangle \geq 2\left(\lambda_{0}-\epsilon\right) f(s) .
\end{aligned}
$$

Thus one has differential inequalities $f^{\prime \prime} \geq \lambda_{0} f$. From this, one can get the desired estimate. This completes the proof.

Let $Y$ be a complete Riemannian four-manifold such that except for a compact subset $K \subset Y, Y \backslash K$ is isometric to $Y(0) \times[0, \infty)$ where $Y(0)$ is a closed manifold. Let us equip a weight function $\tau: Y \rightarrow[0, \infty)$ by $\tau \mid Y(0) \times t=\delta t$. Let $I: L^{2}(Y) \cong L_{\tau}^{2}(Y)$ be the isometry.

Corollary 3.2 24] The corresponding:

$$
P=I^{-1} P_{\tau} I: W^{k+1}\left(Y ; \Lambda^{1}\right) \rightarrow W^{k}\left(Y ; \Lambda^{0} \oplus \Lambda_{+}^{2}\right)
$$

gives a Fredholm map.

Proof This is well known, but for convenience we recall the proof. Let us take a cut-off function $\varphi \mid Y$ with $\varphi \mid Y(0) \times[1, \infty) \equiv 1$, and $\varphi \mid K \equiv 0$. Let us choose any $u \in W^{k+1}\left(Y ; \Lambda^{1}\right)$. Then one may regard $\varphi u \in W^{k+1}\left(Y(0) \times \mathbb{R} ; \Lambda^{1}\right)$. Thus one has the estimate:

$$
\begin{aligned}
|u| W^{k+1} \leq & |\varphi u| W^{k+1}+|(1-\varphi) u| W^{k+1} \\
\leq & C\left\{|\varphi P(u)| W^{k}+|[\varphi, P] u| W^{k}+|(1-\varphi) P u| W^{k}\right. \\
& \left.+|[(1-\varphi), P] u| W^{k}+|(1-\varphi) u| W^{k}\right\} .
\end{aligned}
$$

This shows that $P$ has closed range with finite-dimensional kernel. One has a similar estimate for $P^{*}$. This shows the result. 


\section{D First cohomology}

Let $w=\tau+\mu$ be as in the last paragraph of 3.B.

Lemma 3.4 For a sufficiently small choice of $\delta^{\prime} \ll \delta, d: W_{w}^{k+1}\left(\widetilde{W}_{0} ; \Lambda^{0}\right) \rightarrow$ $W_{w}^{k}\left(\widetilde{W}_{0} ; \Lambda^{1}\right)$ has closed range with ker $d=0$.

Proof Clearly ker $d=0$.

As lemma 3.3, passing through the isometry $I, I^{-1} \circ d \circ I \mid \widetilde{W}_{0}([0, \infty))$ can be expressed as:

$$
\frac{d}{d t}+\left(d,-\frac{\delta}{2}\right): W^{k+1}\left(\widetilde { W } _ { 0 } ( [ 0 , \infty ) ) \rightarrow W ^ { k } \left(\widetilde{W}_{0}\left([0, \infty) ; \Lambda^{0} \oplus \Lambda^{1}\right) .\right.\right.
$$

Clearly this has closed range. One has the same property on $N_{0}([0, \infty))$ and $M_{1}([0, \infty))$.

Now let $H_{i} \subset W^{k+1}\left(\widetilde{W}_{0}\right), i=0,1,2,3$ be closed subsets whose supports lie in $N_{0}([0, \infty)), M_{1}([0, \infty)), \widetilde{W}_{0}([0, \infty))$ and $K$ respectively, where $K \subset \widetilde{W}_{0}$ is some compact subset. Then one may assume $\cup_{i} H_{i}=W^{k+1}\left(\widetilde{W}_{0}\right)$. Now by the open mapping theorem and above, every image $d\left(H_{i}\right)$ has closed range. This completes the proof.

Let us consider $\widetilde{W}_{0}$ and the weight function $w$ on it with the weight constants $\left(\delta, \delta^{\prime}\right)$. Let us recall that we have a bounded complex:

$$
0 \longrightarrow W_{w}^{k+2}\left(\widetilde{W}_{0}\right) \stackrel{d}{\longrightarrow} W_{w}^{k+1}\left(\widetilde{W}_{0} ; \Lambda^{1}\right) \stackrel{d_{+}}{\longrightarrow} W_{w}^{k}\left(\widetilde{W}_{0} ; \Lambda_{+}^{2}\right) \longrightarrow 0
$$

where the weight function is $w=\tau+\mu$.

It is clear ker $d_{0}=0$. Suppose $\omega \in W_{w}^{1}\left(\widetilde{W}_{0} ; \Lambda^{1}\right)$ with $d_{+}(\omega)=0$. Then by integration by parts, one has $0=\int_{\widetilde{W}_{0}}\left|d_{+} \omega\right|^{2}=\frac{1}{2} \int_{\widetilde{W}_{0}}|d \omega|^{2}$. In particular we have $d \omega=0$. The following sublemma shows $H^{1}(\mathrm{AHS})=0$.

Let $W=\widetilde{W}_{0}$ or $\tilde{Y}(S)$ in 2.B. Recall that one has a weight function $\tau$ over $\widetilde{W}_{0}$. There is a natural extension of $\tau$ on $\widetilde{Y}(S)$. Let $\mu: \widetilde{Y}(S) \rightarrow[0, \infty)$ be another weight function defined as $\mu \mid \widetilde{W}_{0}^{n}([-S, S])(x)=\delta^{\prime}(|n|+t(x))$, where $t: \widetilde{W}_{0}^{0}([-S, S]) \rightarrow[0,1]$ satisfies $t \mid N_{0}(S) \equiv 0$ and $t \mid M_{1}(S) \equiv 1$.

Let $w=\tau+\mu$ be a weight function on $W$. In order to make explicit the weight constants, sometimes one uses notations $\tau(\delta)$ and $\mu\left(\delta^{\prime}\right)$. Later one will show that $d: W_{w}^{k+1}(\tilde{Y}(S)) \rightarrow W_{w}^{k}\left(\widetilde{Y}(S) ; \Lambda^{1}\right)$ has closed range. Assuming this, one has the following: 
Sublemma 3.1 Let $f \in C^{\infty}(W)$ satisfies $|d f| L_{w}^{2}(W)<\infty$. Then there are constants $C$ and $k=k(f)$ such that:

$$
|f-k| L_{w}^{2}<\infty .
$$

Proof The situation differs from [27, lemma 5.2], in that on the end, we have non compact slices, $\widetilde{W}_{0}(t), M_{1}(s)$, and $N_{0}(s)$. In order to verify this, one takes two steps. First one sees a weaker version of the sublemma.

Step 1: Let us choose any $\delta_{0}<\delta$ and any $\delta_{0}^{\prime}<\delta^{\prime}$ so that the pair $\left(\delta_{0}, \delta_{0}^{\prime}\right)$ is sufficiently near $\left(\delta, \delta^{\prime}\right)$. Let us put $w=\tau(\delta)+\mu\left(\delta^{\prime}\right)$ and $w^{\prime}=\tau\left(\delta_{0}\right)+\mu\left(\delta_{0}^{\prime}\right)$. Then we claim that $f-k \in L_{w^{\prime}}^{2}$.

By lemma 3.2(1) and lemma 3.4, one has an estimate:

$$
\int_{W} \exp (w)|u|^{2} \leq C \int_{W} \exp (w)|d u|^{2} .
$$

This shows that one has a Hilbert space, which is a completion of $C_{\mathrm{cp}}^{\infty}(W)$ by the norm $|u|^{2}=\int_{W} \exp (w)|d u|^{2}$. Let us take any $f \in C^{\infty}(W)$ with $d f \in L_{w}^{2}$. Using a functional $s: H \rightarrow \mathbb{R}$, by $s(v)=\int_{W} \exp (w)\left(|d v|^{2}-2\langle d v, d f\rangle\right)$, one gets a unique $u=\left(d_{w}^{*} d\right)^{-1}\left(d_{w}^{*} d f\right) \in H$ with $d_{w}^{*} d(f-u)=0$. We show that $g=f-u$ is in $L_{w^{\prime}}^{2}$. From $d g \in L_{w}^{2}$, one has estimates $\int_{W([t, t+1])}|d g|^{2} \leq \exp (-\delta t) \epsilon_{t}$, where $\epsilon_{t} \rightarrow 0$. Notice that $d g$ satisfies the elliptic equation $\left(d_{w}^{*} \oplus d\right) d g=0$. Thus by the local Sobolev embedding and the above estimate, one gets:

$$
\sup _{\widetilde{W}_{0}(t)}|d g| \leq C \exp \left(-\frac{\delta t}{2}\right) \epsilon_{t} .
$$

In particular one has the next estimate:

$$
|g(m, t)| \leq C_{1}+\int_{0}^{t} C_{2} \exp \left(-\frac{\delta s}{2}\right) \epsilon_{s} d s
$$

where $C_{1}=|g(m, 0)|$. This shows that there is a constant $C_{m}$ such that $\lim _{t \rightarrow \infty} \sup _{W(m, t)}\left|g-C_{m}\right|=0$. It is clear that $C_{m}$ is independent of $m$, and we write the same constant by $C$.

Let us make a similar procedure on $s$ direction along $M_{1}(s)$ and $N_{0}(s)$. Then one finds other constants $C^{\prime}$ and $C^{\prime \prime}$ such that $g-C^{\prime} \mid M_{1}(m, s)$ and $g-$ $C^{\prime \prime} \mid N_{0}(m, s)$ vanish at infinity for every $m$. Again it is clear that $C=C^{\prime}=C^{\prime \prime}$.

Now one finds a constant $c$ with $|d g|(x) \leq c \exp \left(-\frac{w(x)}{2}\right)$. Then by integration, one has the inequality:

$$
|g-C|(x) \leq c \exp \left(-\frac{w(x)}{2}\right)
$$


Notice that for any $\alpha>0, w^{\alpha}$ is integrable over $W$. Combining with this and the above inequality, one gets the first claim.

Step 2: For any sufficiently small pair $\left(\delta, \delta^{\prime}\right)$, one has a well-defined first cohomology group $H^{1}\left(\delta, \delta^{\prime}\right)=\operatorname{ker} d \cap L_{w}^{2}\left(W ; \Lambda^{1}\right) / d\left(W_{w}^{1}(W)\right)$. Then one has a natural map:

$$
i: H^{1}\left(\delta, \delta^{\prime}\right) \rightarrow H^{1}\left(\delta_{0}, \delta_{0}^{\prime}\right)
$$

We claim that $i$ is an injection. This is enough for the proof of the sublemma.

Let us put $\Delta\left(\delta, \delta^{\prime}\right)=\left(d_{w}^{*} \oplus d\right)_{w}^{*}\left(d_{w}^{*} \oplus d\right)$ on $L_{w}^{2}\left(W ; \Lambda^{1}\right), w=w\left(\delta, \delta^{\prime}\right)$. Then the spectrum of $\Delta\left(\delta, \delta^{\prime}\right)$ is discrete near 0 . Thus for $\left(\delta_{0}, \delta_{0}^{\prime}\right)$ sufficiently near $\left(\delta, \delta^{\prime}\right)$, one has $\operatorname{dim} H^{1}\left(\delta, \delta^{\prime}\right) \geq \operatorname{dim} H^{1}\left(\delta_{0}, \delta_{0}^{\prime}\right)$.

Suppose these dimensions are different. Then one chooses another $\delta_{1}<\delta_{0}$ and $\delta_{1}^{\prime}<\delta_{0}^{\prime}$. If $\operatorname{dim} H^{1}\left(\delta_{0}, \delta_{0}^{\prime}\right)=\operatorname{dim} H^{1}\left(\delta_{1}, \delta_{1}^{\prime}\right)$, then one puts $\delta_{0}=\delta$ and $\delta^{\prime}=\delta_{0}^{\prime}$, and gets the conclusion. If these dimensions are different, one has $\operatorname{dim} H^{1}\left(\delta_{0}, \delta_{0}^{\prime}\right)>\operatorname{dim} H^{1}\left(\delta_{1}, \delta_{1}^{\prime}\right)$. If they are different, then one must have $\operatorname{dim} H^{1}\left(\delta, \delta^{\prime}\right) \geq 2$. Let us take another $\delta_{2}<\delta_{1}<\delta_{0}$ and $\delta_{2}^{\prime}$ similarly.

One iterates this process until one gets the equality on dimension. If this is not the case, one has an infinite sequence $\left(\delta_{i}, \delta_{i}^{\prime}\right)$. By choice, one may assume that this sequence converges to $\left(\delta_{\infty}, \delta_{\infty}^{\prime}\right)$ with $2 \delta_{0}\left({ }^{\prime}\right)-\delta\left(^{\prime}\right)<\delta_{\infty}\left({ }^{\prime}\right)<\delta\left({ }^{\prime}\right)$. Then $\Delta\left(\delta_{\infty}, \delta_{\infty}^{\prime}\right)$ must have a continuous spectrum near 0 . This is a contradiction. This completes the proof.

Remark 3.1 When $\delta^{\prime}=0$ (no weight in the horizontal direction), one can still find some constant $C$ such that $g-C$ vanishes at infinity. Let us take any $\left(m^{\prime}, t\right) \in \widetilde{W}_{0}^{n}([-S, S]) \subset \widetilde{Y}(S)$. Then we show that if $n$ is sufficiently large, then $\left|g\left(m^{\prime}, t\right)-C_{m}\right|$ is sufficiently small for $C_{m}$ as above. With respect to $n$, let us choose a sufficiently large $T$. Then since one has the estimate $|d g| \tilde{Y}(S)(T) \leq C \exp \left(-\frac{\delta T}{2}\right)$, one may assume $\left|g(m, T)-g\left(m^{\prime}, T\right)\right|$ is sufficiently small. On the other hand, $\left|g\left(m^{\prime}, t\right)-g\left(m^{\prime}, T\right)\right|$ is less than $\epsilon_{n}$, where $\epsilon_{n} \rightarrow 0$ as $n \rightarrow \infty$. Thus $\left|g\left(m^{\prime}, t\right)-C_{m}\right|$ is sufficiently small.

Lemma 3.5 Let $W=\tilde{Y}(S)$. $H^{1}(\delta, 0)$ is naturally isomorphic to $H^{1}\left(\delta, \delta^{\prime}\right)$.

Proof Let $\alpha \in H^{1}(\delta, 0)$ be a harmonic representative. Then from corollary 4.1 below, and by the same method as [27, lemma 5.3], one can verify $\alpha \in$ $L_{w\left(\delta, \delta^{\prime}\right)}^{2}\left(\widetilde{Y}(S) ; \Lambda^{1}\right)$. This completes the proof. 


\section{Fourier-Laplace transforms between open mani- folds}

\section{A Fourier-Laplace transforms}

Our main application is the analysis over $\left(W_{0}, N_{0}, M_{1}\right)=$ kinky handles. Recall that for the simplest kinky handle, $W_{0}$ is diffeomorphic to $S^{1} \times D^{3}$. In this section, one considers $\left(W_{0}=S^{1} \times D^{3}, M_{1}, N_{0}\right)$, where $M_{1}, N_{0}$ are all diffeomorphic to $S^{1} \times D^{2}$, and the embeddings are given as follows. Let us regard $\partial W_{0}=S^{1} \times D_{+}^{2} \cup S^{1} \times D_{-}^{2}$, and consider two knots $\mu \subset S^{1} \times D_{+}^{2}, \gamma \in S^{1} \times D_{-}^{2}$ as follows;

(1) $\mu$ represents a Whitehead link diagram (see [22, page 79, figure 3.3]) and

(2) $\gamma=S^{1} \times 0 \subset S^{1} \times D_{-}^{2}$.

Notice that $\mu$ is null-homotopic, and $\gamma$ represents a generator of $\pi_{1}\left(W_{0}\right)$. Let us take tubular neighborhoods of $\mu$ and $\gamma$ in $S^{1} \times D_{ \pm}^{2}$ respectively. We denote them as $\bar{\mu}$ and $\bar{\gamma}$. Then we choose $\bar{\mu}=M_{1}$ and $\bar{\gamma}=N_{0}$. Let us denote the quotient space by $Y=W_{0} / M_{1} \sim N_{0}$. By a simple calculation, one knows the following:

Sublemma 4.1 $H^{1}(Y ; \mathbb{R})=\mathbb{R}$ and $H^{2}(Y ; \mathbb{R})=H^{3}(Y ; \mathbb{R})=0$

In this section, one introduces the Fourier-Laplace transform over $Y$, which is an open analogue of the one in 27. In order to do this, one will only require the above topological conditions. In particular one can apply the method in this section for cylindrical manifolds $Y$ which includes the simplest Casson handles.

Now by the previous construction, one has a complete Riemannian manifold $\widetilde{W}_{0}$. Let us take infinitely many $\widetilde{W}_{0}$, and for convenience, we enumerate the same $\widetilde{W}_{0}$ as $\widetilde{W}_{0}^{i}, i \in \mathbb{Z}$. Let us choose a sufficiently large $S$, and introduce notations:

$$
\begin{aligned}
& \widetilde{W}_{0}([-S, S])=\widetilde{W}_{0} \backslash\left\{M_{1}([S, \infty)) \cup N_{0}([S, \infty))\right\}, \\
& Y(S)=\widetilde{W}_{0}([-S, S]) / N_{0}(S) \sim M_{1}(S) .
\end{aligned}
$$

One has a natural extension of notation $Y(S)(t)=\widetilde{W}_{0}(t) / N_{0}(S, t) \sim M_{1}(S, t)$, $t \in[0, \infty)$. Recall that one has a weight function $\tau: \widetilde{W}_{0} \rightarrow[0, \infty)$ by $\tau \mid \widetilde{W}_{0}(t)=$ $\delta t$. One can make a natural extension of $\tau$ on $Y(S)$. We will use the same $\tau$ for this. 
Now one has a periodic Riemannian manifold as:

$$
\begin{aligned}
\widetilde{Y}(S)= & \cdots \cup_{M_{1}^{-n}(S) \sim N_{0}^{-n+1}(S)} \widetilde{W}_{0}^{-n+1}([-S, S]) \\
& \cup_{M_{1}^{-n+1}(S) \sim N_{0}^{-n+2}(S)} \widetilde{W}_{0}^{-n+2}([-S, S]) \ldots
\end{aligned}
$$

There is a free and isometric $\mathbb{Z}$-action on $\tilde{Y}(S)$, and we denote the action $1 \in \mathbb{Z}$ by $T$.

Let us take vector bundles $E_{i} \rightarrow Y(S)$ (for $i=1,2$ ) and a differential operator $D$ between them. Then it lifts to the following one:

$$
D: C_{\mathrm{cp}}^{\infty}\left(\widetilde{E}_{1}\right) \rightarrow C_{\mathrm{cp}}^{\infty}\left(\widetilde{E}_{2}\right)
$$

where $\widetilde{E}_{i} \rightarrow \widetilde{Y}(S)$ are the natural lifts.

Let us take any $\psi \in C_{\mathrm{cp}}^{\infty}\left(\widetilde{E}_{1}\right)$ and $z \in \mathbb{C}^{*}$. Then we define the Fourier-Laplace transform of $\psi$ by:

$$
\widehat{\psi}_{z}(\quad)=\Sigma_{n=-\infty}^{\infty} z^{n}\left(T^{n} \psi\right)(\quad) .
$$

Let us define another vector bundle over $Y(S) \times \mathbb{C}^{*}$ as:

$$
E_{1}^{\prime} \equiv\left[\widetilde{E}_{1} \otimes_{\mathbb{R}} \mathbb{C}\right] / \mathbb{Z} \rightarrow Y(S) \times \mathbb{C}^{*}
$$

where $1 \in \mathbb{Z}$ sends $(\rho, \lambda) \in \widetilde{E}_{1} \otimes_{\mathbb{R}} \mathbb{C}$ to $(T \rho, z \lambda)$. One may regard $E_{1}^{\prime}$ as a family of vector bundles $\left\{E_{1}^{\prime}(z)\right\}$ over $Y(S)$, parameterized by $z \in \mathbb{C}^{*}$.

Now by restriction $\widehat{\psi} \mid \widetilde{W}_{0}^{0}([-S, S]), \widehat{\psi}_{z}$ defines a smooth section over $Y(S)$ of $E_{1}^{\prime}(z)$, where $\widetilde{W}_{0}^{0}([-S, S]) \subset \widetilde{Y}(S)$. Thus one may regard $\widehat{\psi}_{z} \in C_{\mathrm{cp}}^{\infty}\left(E_{1}^{\prime}(z)\right)$.

The Fourier-Laplace inversion formula is as follows. Let us take a smooth section $\widehat{\eta} \in C_{\mathrm{cp}}^{\infty}\left(E_{1}^{\prime}\right)$ with $\widehat{\eta}_{z} \in C_{\mathrm{cp}}^{\infty}\left(E_{1}^{\prime}(z)\right)$. Then for any $s \in(0, \infty)$, the following:

$$
T^{n} \eta(x) \equiv \frac{1}{2 \pi i} \int_{|z|=s} z^{-n} \widehat{\eta}_{z}(\pi(x)) \frac{d z}{z}
$$

defines a smooth section over $\widetilde{E}_{1} \rightarrow \tilde{Y}(S)$, where $\pi: \tilde{Y}(S) \rightarrow Y(S)$ is the projection and $x \in \widetilde{W}_{0}^{0}([-S, S])$. By Cauchy's formula, these are inverses of each other, independently of $s$.

Let $D$ be as above. Passing through the Fourier-Laplace transform, one has another differential operator between $\left(E_{0}\right)^{\prime}$ and $\left(E_{1}\right)^{\prime}$ by:

$$
\widehat{D}_{z} \widehat{\psi}_{z} \equiv(\widehat{D \psi})_{z}
$$

Notice that at $z=1,\left(E_{i}\right)^{\prime}, i=0,1$, are isomorphic to $E_{i} \rightarrow Y(S)$ respectively. In fact every $\left(E_{i}\right)^{\prime}(z)$ is isomorphic to $E_{i}$ as follows. Let us take 
$t: \widetilde{W}_{0}([-S, S]) \rightarrow[0,1]$, a smooth map such that $t \equiv 0$ near $N_{0}(S)$, and $t \equiv 1$ near $M_{1}(S)$. By taking $[0, \infty) \subset \mathbb{C}$ as a branched line, one may define $\log z$. Let us put $z^{t} \equiv \exp (t \log z)$, and consider $z^{t} \widehat{\psi}_{z}$. This gives a $\mathbb{C}$-valued section over $E_{0}^{\prime}$. One calculates $\widehat{D}_{z}$ in terms of this identification. The result is as:

$$
\widehat{D}(z)=D+z^{t}\left[D, z^{-t}\right]: C_{\mathrm{cp}}^{\infty}\left(E_{0}\right) \rightarrow C_{\mathrm{cp}}^{\infty}\left(E_{1}\right)
$$

\section{B Elliptic complexes over periodic covers}

Let $\left\{E_{i}, D_{i}\right\}_{i=0,1,2}$ be an elliptic complex over $Y(S)$. It is clear that it gives a bounded one as:

$$
0 \longrightarrow W_{\tau}^{k+2}\left(\widetilde{E}_{0}\right) \stackrel{D_{0}}{\longrightarrow} W_{\tau}^{k+1}\left(\widetilde{E}_{1}\right) \stackrel{D_{1}}{\longrightarrow} W_{\tau}^{k}\left(\widetilde{E}_{2}\right) \longrightarrow 0 .
$$

Proposition 4.1 The above $D_{i}$ is an acyclic Fredholm complex, ie all cohomologies $H^{i}$ vanish, if for all $z \in C=\left\{w \in \mathbb{C}^{*} ;|w|=1\right\},\left\{\widehat{D}_{z}^{i}: W_{\tau}^{k+i} \rightarrow\right.$ $\left.W_{\tau}^{k+i-1}\right\}$ are also so.

Proof For any $u \in W_{\tau}^{k}\left(\widetilde{Y}(S) ; \widetilde{E}_{i}\right)$, $\widehat{u}_{z}$ has the following property; it lies in $W_{\tau}^{k}\left(\left(E_{i}\right)_{z} ; Y(S)_{z}\right)$ for all $z$ with $0<|z| \leq 1$.

When $|z|=1$, the inner product on $E_{i}$ has a natural extension of a Hermitian metric on $E_{i}(z)$. Recall that $D_{i}: W_{\tau}^{k+1}\left(Y(S) ; E_{i}(z)\right) \rightarrow W_{\tau}^{k}\left(Y(S) ; E_{i+1}(z)\right)$ has closed range. Let $\left(D_{i}\right)_{\tau}^{*}$ be the formal adjoint operator. This differential operator is defined independently of $z$, with $|z|=1$ over $E_{i}(z)$. By the assumption, one has an isomorphism:

$$
D=D_{0} \oplus\left(D_{1}\right)_{\tau}^{*}: W_{\tau}^{k+1}\left(Y(S) ; E_{0}(z) \oplus E_{2}(z)\right) \rightarrow W_{\tau}^{k}\left(Y(S) ; E_{1}(z)\right) .
$$

We denote by $D^{-1}$ its inverse. On the other hand one has a natural lift of $D$ as:

$$
D: W_{w}^{k+1}\left(\widetilde{Y}(S) ; \widetilde{E}_{0} \oplus \widetilde{E}_{2}\right) \rightarrow W_{w}^{k}\left(\widetilde{Y}(S) ; \widetilde{E}_{1}\right) .
$$

Here we show that there is another bounded operator:

$$
R: W_{w}^{k}\left(\widetilde{Y}(S) ; \widetilde{E}_{1}\right) \rightarrow W_{w}^{k+1}\left(\widetilde{Y}(S) ; \widetilde{E}_{0} \oplus \widetilde{E}_{2}\right)
$$

with $R \circ D$ is the identity. From this, one knows that $D$ has closed range. In particular $D_{0}$ has closed range.

Next one proceeds similarly for $D_{\tau}^{*}=\left(D_{0}\right)_{\tau}^{*} \oplus D_{1}$. Then one also knows that its lift $D_{\tau}^{*}$ has closed range. Clearly it follows that $D_{1}$ has also closed range. 
Now let us take $\psi \in W_{w}^{k+1}\left(\widetilde{Y}(S) ; \widetilde{E}_{0} \oplus \widetilde{E}_{2}\right)$. Then one considers $b(\psi)=$ $D^{-1}(\widehat{\psi}) \in W_{\tau}^{k+1}\left(Y(S) ; E_{1}(z)\right)$. This element also has the property in the first paragraph of the proof.

Then one defines $R(\psi) \in W_{w}^{k+1}\left(\widetilde{Y}(S) ; \widetilde{E}_{1}\right)$ by:

$$
T^{n} R(\psi) \mid \widetilde{W}_{0}^{0}([-S, S])(x)=\frac{1}{2 \pi i} \int_{C} z^{-n}\left(D^{-1}(\widehat{\psi})(\pi(x))\right) \frac{d z}{z} .
$$

We claim that this assignment:

$$
R: W_{w}^{k}\left(\widetilde{Y}(S) ; \widetilde{E}_{1}\right) \rightarrow W_{w}^{k+1}\left(\widetilde{Y}(S) ; \widetilde{E}_{0} \oplus \widetilde{E}_{2}\right)
$$

gives a bounded operator. Then by the definition of $D_{i}$ over $Y(S)$, it satisfies $R \circ D=1$.

Let us put $C=\{z=\exp (i \theta) \in \mathbb{C}\}, \psi_{n} \mid \widetilde{W}_{0}^{0}([-S, S])=T^{n}(\psi)$. By regarding $b(\psi)_{z} \in W_{\tau}^{k+1}\left(\widetilde{W}_{0}^{0}([-S, S])\right)$, one has a Fourier expansion $b(\psi)=\Sigma_{n} z^{n} \varphi_{n}$, $\varphi_{n+1}\left|N_{0}(S)=\varphi_{n}\right| M_{1}(S)$. Notice the equality $D\left(\varphi_{n}\right)=\psi_{n}$. One can regard the value of the inner product $\langle b(\psi), b(\psi)\rangle \mid W_{\tau}^{k}\left(Y(S) ; E_{*}(z)\right)$ as a real-valued function of $\theta \in[0,2 \pi]$. Then one has the equality:

$$
\begin{aligned}
& \frac{1}{2 \pi} \int_{0}^{2 \pi} d \theta|b(\psi)|^{2} W_{\tau}^{k+1}\left(Y(S) ; E_{*}(z)\right)= \\
& \left.\frac{1}{2 \pi} \int_{0}^{2 \pi} d \theta \Sigma_{n, m} \exp (i \theta(n-m))\left\langle\varphi_{n}, \varphi_{m}\right\rangle\left|W_{\tau}^{k+1}\left(\widetilde{W}_{0}^{0}([-S, S])\right)=\Sigma_{n}\right| \varphi_{n}\right|^{2} W_{\tau}^{k+1} .
\end{aligned}
$$

Combining with the inequality $\left(D^{-1}\right.$ are bounded operators for all $z \in \mathbb{C}$ ) $|b(\psi)| W_{\tau}^{k+1}\left(Y(S) ; E_{*}(z)\right) \leq C|\widehat{\psi}| W_{\tau}^{k}$ for every $\theta$, one gets the estimate:

$$
\Sigma_{n}\left|\varphi_{n}\right| W_{\tau}^{k+1}\left(\widetilde{W}_{0}^{0}([-S, S])\right) \leq C \Sigma_{n}\left|\psi_{n}\right| W_{\tau}^{k}\left(\widetilde{W}_{0}^{0}([-S, S])\right) .
$$

From the equality, $T^{n}(\psi)=\psi_{n}$, one has the desired estimate:

$$
\begin{gathered}
|R(\psi)|^{2} W_{\tau}^{k+1}\left(\widetilde{Y}(S) ; \widetilde{E}_{*}\right)=\Sigma_{n}\left|\varphi_{n}\right|^{2} W_{\tau}^{k+1}\left(\widetilde{W}_{0}^{0}([-S, S])\right) \leq C \Sigma_{n}\left|D\left(\varphi_{n}\right)\right|^{2} W_{\tau}^{k} \\
=C \Sigma_{n}\left|T^{n}(\psi)\right|^{2} W_{\tau}^{k}\left(\widetilde{W}_{0}^{0}([-S, S])\right)=C|\psi|^{2} W_{\tau}^{k} .
\end{gathered}
$$

This completes the proof.

\section{C Computation of parametrized cohomology groups}

In 4.C, one will verify the assumption in proposition 4.1 .

Sublemma 4.2 $D_{i}: W_{\tau}^{k+1}\left(Y(S) ; E_{i}(z)\right) \rightarrow W_{\tau}^{k}\left(Y(S) ; E_{i+1}(z)\right)$ has closed range. 
Proof It is true for $z=1$. Recall $Y(S)=\widetilde{W}_{0}([-S, S]) / N_{0}(S) \sim M_{1}(S)$. Let $H_{i} \subset W_{\tau}^{k+1}\left(Y(S) ; E_{i}(z)\right)$ be closed subsets satisfying:

(1) $H_{1} \cup H_{2}=W_{\tau}^{k+1}\left(Y(S) ; E_{i}(z)\right)$ and

(2) $\operatorname{Supp} H_{1} \subset \widetilde{W}_{0}([-(S-1), S-1])$ and $\operatorname{Supp} H_{2} \subset N_{0}([-S,-(S-1)]) \cup$ $M_{1}([S-1, S])$.

By regarding $H_{1} \subset W_{\tau}^{k+1}\left(Y(S) ; E_{i}(1)\right)$, it follows that $D_{i}\left(H_{1}\right)$ is a closed subspace of $W_{\tau}^{k}\left(Y(S) ; E_{i+1}(z)\right)$. Let $u \in H_{2}$. Then one may associate $\widetilde{u} \in$ $W_{\tau}^{k+1}\left(Y(S) ; E_{i}(1)\right)$ by $\widetilde{u} \mid N_{0}([-S,-(S-1)])=u$ and $\widetilde{u} \mid M_{1}([S-1, S])=z^{-1} u$. Clearly $D_{i}\left(H_{2}\right)=D_{i}\left(\widetilde{H}_{2}\right)$ is a closed subspace of $W_{\tau}^{k}\left(Y(S) ; E_{i+1}(1)\right)$. Since this assignment is isometric, it follows that $\mathrm{H}_{2}$ is also a closed subspace of $W_{\tau}^{k}\left(Y(S) ; E_{i+1}(z)\right)$.

This completes the proof.

Proposition 4.2 Let $\left\{D_{*}\right\}$ be an AHS complex. Suppose it gives a Fredholm complex and all the cohomology groups vanish, $H^{i}\left(W_{\tau}^{k}\left(Y(S) ; \Lambda^{*}\right) ; D_{*}\right)=0$, $i=0,1,2$. Then for $C(1)=\{z:|z|=1\}$, one has $H^{*}\left(W_{\tau}^{k}\left(Y(S) ; \Lambda^{*}\right) ;\left(D_{*}\right)_{z}\right)=$ 0 for all $z \in C(1)$ and $i=0,1,2$.

Proof The first part is essentially [27, page 390]. Let $u \in W_{\tau}^{k}\left(Y(S) ; \Lambda_{z}^{*}\right)$ with $d_{0}(u)=0$. Then passing through the identification $z^{t}: \Lambda_{z}^{0} \cong \Lambda^{0}$, one has $d\left(z^{-t} u\right)=0$ where $z^{-t} u \in W_{\tau}^{k}\left(Y(S) ; \Lambda^{0}\right)$. Clearly this shows $u=0$.

Next let us take $\alpha \in W_{\tau}^{k}\left(Y(S) ; \Lambda_{z}^{1}\right)$ with $d_{+}(\alpha)=0, d_{\tau}^{*}(\alpha)=0$. Then by integration by parts, one has $d(\alpha)=0$. $\alpha$ is zero in $H^{1}\left(\widetilde{W}_{0} ; \mathbb{R}\right)$ (see the proof of lemma 5.1). Thus one may express $\alpha=d(f)$ on $\widetilde{W}_{0}$ for $f \in C^{\infty}\left(\widetilde{W}_{0}([-S, S])\right)$ and $d_{\tau}^{*} d(f)=0$. Let $i: N_{0}(S) \cong M_{1}(S)$ be the identification. Then one has $i^{*}(f)=z f+$ const. If one takes $f$ so that it vanishes at infinity, then const $=0$. Thus one has the equality:

$$
0=\int_{\widetilde{W}_{0}([-S, S])} \exp (\tau)\left\langle f, d_{\tau}^{*} d(f)\right\rangle v o l=-\int \exp (\tau)|d f|^{2} .
$$

This shows $d f=0$. Let $O \subset C(1)$ be the subset satisfying a property that any $\alpha \in W_{\tau}^{k}\left(Y(S) ; \Lambda_{z}^{1}\right)$ with $d_{+}(\alpha)=0$ satisfies $d \alpha=0$. The above implies $O$ is non-empty, open and closed in $C(1)$. Thus $H^{*}\left(W_{\tau}^{k}\left(Y(S) ; \Lambda^{*}\right) ;\left(D_{*}\right)_{z}\right)=0$, $*=0,1$ for all $z \in C(1)$.

Now let us put $D=\left(D_{0}\right)_{\tau}^{*} \oplus D_{1}$, and consider $\Delta=D_{\tau}^{*} D$ on $L_{\tau}^{2}\left(Y(S) ; \Lambda^{1}(z)\right)$. Then there is a constant $\lambda>0$ with $\lambda_{z}>\lambda$, where $\lambda_{z}$ is the bottom of $\Delta$. Suppose $H^{2} \neq 0$ at $z=\exp \left(i \theta_{0}\right)$, but $H^{2}=0$ for all $z=\exp (i \theta), 0 \leq \theta<\theta_{0}$. 
Then by choosing some $\theta$, it follows that there exists $u \in W_{\tau}^{1}\left(Y(S) ; \Lambda_{z}^{2}\right), z=$ $\exp (i \theta)$ with $(1)|u| W_{\tau}^{1}=1$ and $(2)\left|\left(D_{1}\right)_{\tau}^{*}(u)\right| L_{\tau}^{2}<\epsilon$.

Since $D_{1}$ is surjective, one finds $\alpha \in W_{\tau}^{2}\left(Y(S) ; \Lambda_{z}^{1}\right)$ with $D_{1}(\alpha)=u$ and $\left(D_{0}\right)_{\tau}^{*}(\alpha)=0$. Then there is a constant $C$ independent of $z$ such that:

$$
|u| W_{\tau}^{1} \leq C|\alpha| W_{\tau}^{2} \leq C\left|\left(D_{1}\right)_{\tau}^{*} D_{1}(\alpha)\right| L_{\tau}^{2} .
$$

This is a contradiction. This shows that the second cohomology also vanishes for all $z \in C(1)$. This completes the proof.

\section{D Computation of cohomology groups with $z=1$}

Now we compute the cohomology $H^{i}\left(W_{\tau}^{k}\left(Y(S) ; \Lambda^{*}\right) ; D_{*}\right)$ of the AHS complex for the simplest kinky handles $\left(W_{0}, N_{0}, M_{1}\right)$. Notice that the end of $Y(S)$ is isometric to $M \times[0, \infty)$ for a compact Riemannian three manifold $M$. We will denote $M_{t}=M \times\{t\} \subset Y(S)$.

Proposition 4.3 Let $Y(S)$ be as above. Then for all $i=0,1,2$, the cohomologies $H^{i}\left(W_{\tau}^{k}\left(Y(S) ; \Lambda^{*}\right) ; D_{*}\right)$ vanish.

Proof Clearly $H^{0}$ (AHS) $=0$. Let us consider $H^{1}$ (AHS). Let us take any representative $u$. By integration by parts, one finds $d u=0$. One may express $u=w+f d t$ around the end $Y(S) \cong M \times[0, \infty)$, where $w$ does not contain $d t$ component. Then through this isometry, one considers $w_{t} \in \Lambda^{1}(M)$ for $t \in[0, \infty)$. Let $d_{3}$ be the differential over $M$. Then for every $t$, one has $d_{3} w_{t}=0$. Thus one may express $w_{t}=\Sigma_{i} g_{i}(t) \alpha_{i}+d \mu_{t}$, where $\left\{\alpha_{i}\right\}_{i}$ consists of the orthogonal basis of $H^{1}(M ; \mathbb{R})$. Here one may assume $d_{3}^{*}\left(\alpha_{i}\right)=0$. By taking into account of $d t$ component of $d u$, one has the following equation:

$$
\Sigma_{i} g_{i}(t)^{\prime} \alpha_{i}+d_{3} \mu(t)^{\prime}=d_{3} f_{t} .
$$

This shows $g_{i}(t)^{\prime} \equiv 0$. Since $u \in L_{\tau}^{2}$, one concludes $g_{i}(t) \equiv 0$. Then one has another equality, $f_{t}=\mu(t)^{\prime}+c(t)$, where $c(t)$ are constants depending on $t$. Thus one gets $u=d_{3} \mu_{t}+\left(\mu_{t}^{\prime}+c(t)\right) d t$.

Sublemma 4.3 If $d_{3} \mu_{t} \in W_{\tau}^{k}$, then for a smooth family of constants $d(t)$, one has $\mu_{t}-d(t) \in W_{\tau}^{k}$.

Proof of sublemma Let us put $d(t)=\left(v_{0} l_{M}\right)^{-1} \int_{M} \mu_{t} v o l$. Then we show that this family is the desired one. Notice that one has the following bound: $\left|d_{3} \mu_{t}\right| L_{\tau}^{2} \geq C\left|\mu_{t}-d(t)\right| L_{\tau}^{2}$ for some positive constant $C$. In particular one has 
$\mu_{t}-d(t) \in L_{\tau}^{2}$. Notice that one has the equality $\mu_{t}-d(t)=\left(\Delta_{0}\right)^{-1} d^{*} d\left(\mu_{t}-d(t)\right)$, and $\left(\Delta_{0}\right)^{-1} d^{*}$ is a translation invariant bounded operator. Thus we get:

$$
\frac{\mu_{t}-d(t)}{d t}=\frac{d}{d t}\left[\left(\Delta_{0}\right)^{-1} d_{3}^{*} d_{3}\left(\mu_{t}-d(t)\right)\right]=\left(\Delta_{0}\right)^{-1} d^{*} \frac{d}{d t}\left[d_{3} \mu_{t}\right]
$$

From the last term, one sees that $\frac{\mu_{t}-d(t)}{d t} \in L_{\tau}^{2}$. By a similar consideration, one gets the result. This completes the proof of the sublemma.

Proof of proposition (continued) Then by replacing $\mu_{t}$ by $\mu_{t}-d(t)$, one may assume $\mu_{t} \in W_{\tau}^{k}$. Thus $d \mu_{t}=d_{3} \mu_{t}+\mu_{t}^{\prime} d t \in W_{\tau}^{k-1}$. On the other hand, let us consider the equality $f_{t}=\mu_{t}^{\prime}+c(t)$. Then it follows $c(t) \in W_{\tau}^{k-1}$. Let us put $C(t)=\int_{0}^{t} c(s) d s-\int_{0}^{\infty} c(t)$. Then by [27, lemma 5.2] (see sublemma 3.1), one finds that $C(t) \in W_{\tau}^{k}$, and $d C(t)=c(t)$. In particular we have $u \mid$ end $Y(S)=d f, f \in W_{\tau}^{k}$. Then using a cut-off function, one may represent $u$ by a compactly supported smooth 1 form, which is itself exact by a compactly supported smooth function, since $H_{\mathrm{cp}}^{1}(Y(S) ; \mathbb{R})=0$. This shows $H^{1}(\mathrm{AHS})=$ 0 .

Next we consider $H^{2}$ (AHS). Let us take a representative $u \in H^{2}(\mathrm{AHS})$. One may choose $u$ so that it satisfies $d\left(e^{\tau} u\right)=e^{-\tau} d\left(u e^{\tau}\right)=0$. Since $H^{2}(Y(S) ; \mathbb{R})=$ 0 , one may express $e^{\tau} u=d \mu, \mu \in C^{\infty}\left(\Lambda^{1}\right)$. Let us denote $\mu \mid$ end $Y(S)=$ $\beta+f d t$, where $\beta$ does not contain $d t$ component. Then we have the following relation:

$$
d \mu \mid \text { end } Y(S)=d_{3} \beta_{t}+\left(d_{3} f-\beta_{t}^{\prime}\right) d t=d_{3} \beta_{t}+*_{3} d_{3} \beta_{t} \wedge d t
$$

where $*_{3}$ is the star operator over $M$. Let us decompose $\beta_{t}=\beta_{t}^{1}+\beta_{t}^{2}$, where $\beta_{t}^{1}\left(\beta_{t}^{2}\right)$ does (not) consists of a closed form over $M$. Then from the last two terms, one finds $d_{3} f_{t}=\left(\beta_{t}^{1}\right)^{\prime}$. In particular one may represent:

$$
e^{w} u \mid \text { end } Y(S)=d \beta_{t}^{2}=d_{3} \beta_{t}^{2}-\left(\beta_{t}^{2}\right)^{\prime} \wedge d t=d_{3} \beta_{t}^{2}+*_{3} d_{3} \beta_{t}^{2} \wedge d t
$$

By the decomposition, one finds a positive constant $C$ such that:

$$
\left|d_{3} \beta_{t}^{2}\right| W^{k-1}\left(M_{t}\right) \geq C\left|\beta_{t}^{2}\right| W^{k}\left(M_{t}\right) .
$$

Now we have the next relations (put $\mu=\beta_{t}^{2}$ on the end):

$$
e^{\tau} u=d \mu, \quad\left|\mu_{t}\right| W^{k}\left(M_{t}\right) \leq C\left|e^{\tau} u\right| W^{k-1}\left(M_{t}\right)
$$

Sublemma 4.4 If $\delta>0$ is sufficiently small with respect to $S$, then $e^{\tau} u \in$ $L^{2}\left(Y(S) ; \Lambda_{+}^{2}\right)$. 
Proof of sublemma Let us consider $e^{\tau} u \mid$ end $=d \mu$. One may assume that for every $t, \mu_{t} \in C^{\infty}\left(\Lambda^{1}\left(M_{t}\right)\right)$ lies on the orthogonal complement of ker $d$. Then $*_{3} d$ is invertible on $(\operatorname{ker} d)^{\perp}$ by corollary 3.1. Moreover it is self-adjoint with respect to the $L^{2}$ inner product. Since $\mu$ satisfies the equation, $\left(\frac{\partial}{\partial t}+*_{3} d_{3}\right) \mu=$ 0 , one has the exponential decay estimate for $\mu$. More precisely there exist constants $C>0, \lambda_{0}>0$ which are independent of $\delta$, such that:

$$
|\mu| L^{2}\left(M_{t}\right) \leq \exp \left(-\lambda_{0} t\right) \sup \left\{|\mu| L^{2}\left(M_{s}\right) ; 0 \leq s \leq 2 t\right\} .
$$

Notice that a priori, $\mu$ satisfies the following bound of its growth $|\mu| L^{2}\left(M_{t}\right) \leq$ $C \exp \left(t \frac{\delta}{2}\right)|u| L^{2}\left(M_{t}\right)$. Combining these estimates, one gets the exponential estimate for $\mu$. Then one has also the exponential decay estimate for $d \mu$ on the end. This completes the proof of the sublemma.

Proof of proposition (continued) Let $\varphi_{t}: Y(S) \rightarrow[0,1]$ be a cut-off function such that $\varphi|M \times[t+1, \infty) \equiv 0, \varphi|(M \times[t, \infty))^{c} \equiv 1$. Let us fix a large $S$, and let $\delta>0$ satisfy the above condition. By the above estimate on $W^{k}$ norm, one finds $\varphi_{t} \mu \in L^{2}\left(Y(S) ; \Lambda_{+}^{2}\right)$ for all $t$. Then for any small $\epsilon>0$, one finds a large $t$ so that:

$$
\int\left|e^{w} u\right|^{2}=\left.\left|\int d\left(\varphi_{t} \mu\right) \wedge d\left(\varphi_{t} \mu\right)+\int\right| e^{w} u\right|^{2} \mid<\epsilon .
$$

This shows $u \equiv 0$, and we have shown $H^{2}(\mathrm{AHS})=0$ for this pair $(\delta, S)$. This completes the proof of the proposition.

Remark 4.1 Suppose for all $\delta^{\prime} \in\left[\delta, \delta_{0}\right]$, the differential of the AHS complex has closed range, where $\delta$ is sufficiently small. Then $H^{*}(\mathrm{AHS})$ also vanishes for all $\delta^{\prime}$. This is seen as follows. Notice that for any choice of the constants, one knows $H^{0}(\mathrm{AHS})=H^{1}(\mathrm{AHS})=0$. We want to calculate $H^{2}(\mathrm{AHS})$ when we vary $\delta^{\prime}>0$. Let us take an isomorphism:

$$
I: W_{\tau(\delta)}^{*}\left(Y(S) ; \Lambda^{*}\right) \cong W_{\tau\left(\delta^{\prime}\right)}^{*}\left(Y(S) ; \Lambda^{*}\right)
$$

where we denote $\tau(\delta)$ to express the weight constant. Passing through $I$, one has $\left\{W_{\tau(\delta)}^{*}\left(Y(S) ; \Lambda^{*}\right) ; I^{-1} \circ d_{*} \circ I\right\}$, a continuous family of Fredholm complexes. In particular, the indices of these complexes are invariant. Since for all cases, one has $H^{0}(\mathrm{AHS})=H^{1}(\mathrm{AHS})=0$, one concludes $H^{2}(\mathrm{AHS})=0$.

Remark 4.2 Suppose a cylindrical four-manifold $Y$ has nonzero $H^{2}(Y ; \mathbb{R})$. Then in this case, one has a bound:

$$
\operatorname{dim} H^{2}(\mathrm{AHS}) \leq 2 \operatorname{dim} H^{2}(Y ; \mathbb{R}) .
$$

This directly follows from the proof of proposition 4.3. 
Corollary 4.1 Let us choose a sufficiently small $\delta>0$. Then one has an invertible operator:

$$
P_{w}: W_{w}^{k+1}\left(\tilde{Y}(S) ; \Lambda^{1}\right) \cong W_{w}^{k}\left(\tilde{Y}(S) ; \Lambda^{0} \oplus \Lambda_{+}^{2}\right) .
$$

\section{An asymptotic method to compute cohomology}

\section{A $\quad P_{w}$ over $Y(S, 2)$}

Let $\left(W_{0}, N_{0}, M_{1}\right)$ be a simplest kinky handle, and $\widetilde{W}_{0}$ be the Riemannian manifold constructed before. In the previous section, one has the invertible operator:

$$
P_{\tau}: W_{\tau}^{k+1}\left(Y(S) ; \Lambda^{1}\right) \rightarrow W_{\tau}^{k}\left(Y(S) ; \Lambda^{0} \oplus \Lambda_{+}^{2}\right) .
$$

By the Fourier-Laplace transform, one gets an invertible Fredholm $P_{\tau}$ over $\widetilde{Y}(S)$ where:

$$
\tilde{Y}(S)=\cdots \cup \widetilde{W}_{0}^{i-1}([-S, S]) \cup \widetilde{W}_{0}^{i}([-S, S]) \cup \ldots \quad i \in \mathbb{Z} .
$$

Let $T_{2}$ be the periodic tree as $\mathbb{R} \cup_{n \in \mathbb{Z}} \mathbb{R}_{0}$, where $\mathbb{R}_{0}$ is half the real line. The aim here is to show that $P_{\tau}$ is invertible over the periodic cover of $Y(S, 2)$ defined below which corresponds to $T_{2}$. Let $\left(W_{2}, N_{0}, M_{1}, M_{2}\right)$ be a kinky handle with two kinks, and $\widetilde{W}_{2}$ be the corresponding complete Riemannian manifold. Thus one has three ends in the horizontal direction, $N_{0}([0, \infty)), M_{j}([0, \infty)), j=1,2$. Let us choose a large $S$, and put:

$$
\begin{aligned}
& \tilde{Y}(S)_{0}=\widetilde{W}_{0}^{1}((-\infty, S]) \cup \widetilde{W}_{0}^{2}([-S, S]) \cup \ldots, \\
& Y(S, 2)^{\prime}=\widetilde{W}_{2} \backslash N_{0}([S, \infty)) \cup M_{1}([S, \infty)) / N_{0}(S) \sim M_{1}(S), \\
& Y(S, 2)=Y(S, 2)^{\prime} \backslash M_{2}([S, \infty)) \cup \widetilde{Y}(S)_{0} \backslash N_{0}([S, \infty)) .
\end{aligned}
$$

One may equip a weight function $\tau$ on $Y(S, 2)$ as before.

Lemma $5.1 d_{*}: W_{\tau}^{k+1}\left(Y(S, 2) ; \Lambda^{*}\right) \rightarrow W_{\tau}^{k}\left(Y(S, 2) ; \Lambda^{*+1}\right)$ has closed range with $H^{0}(\mathrm{AHS})=H^{1}(\mathrm{AHS})=0$.

Proof Closedness follows from the excision method used before. Let us take $\left(W_{s}=S^{1} \times D^{3}, N_{0}=S^{1} \times D^{2}\right)$, where $N_{0}$ represents a generator of $\pi_{1}\left(W_{s}\right)$. By the construction in 2.A, one can get a complete Riemannian manifold $\widetilde{W}_{s}$ with one end $N_{0}([0, \infty))$ along the horizontal direction. Let us put:

$$
Y(S, 2)(0)=Y(S, 2)^{\prime} \backslash M_{2}([S, \infty)) \cup \widetilde{W}_{s} \backslash N_{0}([S, \infty)) .
$$


Notice that $Y(S, 2)(0)$ is diffeomorphic to $Y(S, 2)^{\prime}$, but Riemannian metrics are different. $(Y(S, 2)(0)$ has cylindrical end.) By corollary 3.2, the differential of the AHS complex has closed range.

Let $H_{i} \subset W_{\tau}^{k+1}\left(Y(S, 2) ; \Lambda^{*}\right)$ be closed subsets with:

(1) $H_{1} \cup H_{2}=W_{\tau}^{k+1}\left(Y(S, 2) ; \Lambda^{*}\right)$,

(2) Supp $H_{1} \subset Y(S, 2)^{\prime} \backslash M_{2}([S, \infty))$ and Supp $H_{2} \subset \tilde{Y}(S)_{0} \backslash N_{0}([S-1, \infty))$.

One regards $H_{1} \subset W_{\tau}^{k+1}\left(Y(S, 2)(0) ; \Lambda^{*}\right)$ and $H_{2} \subset W_{\tau}^{k+1}\left(\widetilde{Y}(S) ; \Lambda^{*}\right)$. From this, it follows that $d_{*}\left(H_{i}\right)$ are both closed subspace of $W_{\tau}^{k}\left(Y(S, 2) ; \Lambda^{*+1}\right)$.

Let us see $\operatorname{ker} P_{w}=0$. $\operatorname{ker} P_{w}$ is isomorphic to the first cohomology $H^{1}$ (AHS). One can easily check $H^{1}(Y(S, 2) ; \mathbb{R})=\mathbb{R}$. Let us take a nonzero element $w$. Then this has the property $\langle w, C\rangle \neq 0$ for any loop $C$ representing a generator of $\pi_{1}(Y(S, 2))$. One may choose $C$ sufficiently near to infinity while having a bounded length.

Let us take $u \in H^{1}(\mathrm{AHS})$, and consider its class $[u] \in H^{1}(Y(S, 2) ; \mathbb{R})$. It follows $[u]=0$, since one can make $\langle u, C\rangle$ arbitrarily small by choosing $C$ as above. Thus one may express $u=d f$ for $f \in C^{\infty}(Y(S, 2))$. One may assume $f \in L_{w}^{2}(Y(S, 2))$, by subtracting some constant. This shows $u=0$. Thus we have shown the result.

\section{B Some continuity of $H^{1}(\mathrm{AHS})$}

Let us introduce another weight function:

$$
\mu: \tilde{Y}(S) \rightarrow[0, \infty)
$$

by $\mu\left|\widetilde{W}_{n}(s \leq S)(x)=\right| n \mid+t(x)$. We choose another small constant $\delta^{\prime}$ (we take it so that $\delta^{\prime} \ll \delta$, where $\delta$ is the weight constant for $\tau$ ). Then we introduce another weight $w=\delta^{\prime} \mu+\tau$ and weighted Sobolev $k$ spaces $W_{w}^{k}$, where:

$$
\left(|u| W_{w}^{k}\right)^{2}=\int_{\widetilde{Y}} \exp \left(\delta^{\prime} \mu+\tau\right)\left(\Sigma_{l=0}^{k}\left|\nabla^{l} u\right|^{2}\right) .
$$

Since one knows $H^{*}(\mathrm{AHS})=0$ over $\tilde{Y}(S)$, it follows that there exists a positive constant $C>0$ such that $\left|P_{w}\right|,\left|\left(P_{w}\right)_{w}^{*}\right| \geq C$, for any small $\delta^{\prime} \in\left[0, \delta_{0}^{\prime}\right]$.

Let $Y$ be a complete Riemannian manifold of bounded geometry. Let us take a family of smooth maps:

$$
w\left(\delta^{\prime}\right): Y \rightarrow[0, \infty), \quad w\left(\delta^{\prime \prime}\right)(x) \leq w\left(\delta^{\prime}\right)(x), \quad \delta^{\prime \prime} \leq \delta^{\prime} \in[0, \delta], \quad x \in Y .
$$

Suppose that for all $w\left(\delta^{\prime}\right)$, the corresponding AHS complex between weighted Sobolev spaces are Fredholm. In any case it is immediate to see $H^{0}(\mathrm{AHS})=0$. Here one has some continuity property as: 
Lemma 5.2 $H^{1}(\mathrm{AHS})=0$ for $\delta^{\prime}=0$ when $H^{1}(\mathrm{AHS})=0$ for $\delta^{\prime}>0$.

Proof Let us denote $\operatorname{ker}\left(d_{+}\right)_{w\left(\delta^{\prime}\right)}^{*}=H^{2}\left(\delta^{\prime}\right)$ (the second cohomology $H^{2}$ (AHS) when it is defined). Let us choose sufficiently small constants:

$$
\delta \gg \delta^{\prime}>\delta^{\prime \prime} \geq 0 .
$$

Then one has $H^{2}\left(\delta^{\prime \prime}\right) \cong H^{2}\left(\delta^{\prime}\right)$. This follows from that by varying these weights from $w\left(\delta^{\prime}\right)$ to $w\left(\delta^{\prime \prime}\right)$, one gets a family of Fredholm complexes between the weighted Sobolev spaces (these spaces also vary with respect to the deformation of the weights). For every value of weights, one has $H^{0}(\mathrm{AHS})=H^{1}(\mathrm{AHS})=0$. Thus one gets the above statement.

Sublemma 5.1 If $H^{2}\left(\delta^{\prime \prime}\right) \neq 0$, then one has also $H^{2}\left(\delta^{\prime}\right) \neq 0$.

Proof of sublemma Let us denote $w=w\left(\delta^{\prime}\right)$ and $w^{\prime}=w\left(\delta^{\prime \prime}\right)$. Let us take $v \in L_{w^{\prime}}^{2}$ with $d^{*}\left(\exp \left(w^{\prime}\right) v\right)=0$. Then one puts $u=\exp \left(-w+w^{\prime}\right) v$. One may assume $u \in L_{w}^{2}$. Since $u$ satisfies $d^{*}(\exp (w) u)=0$, one gets the result.

Proof of lemma (continued) Recall that by putting $w=w\left(\delta^{\prime}\right)$, one also has a Fredholm complex $\left\{W_{w}^{*}\left(Y(S, 2) ; \Lambda^{*}\right), d^{*}\right\}$. Then by varying a parameter $\delta^{\prime} \in[0, \delta]$, one has a family of Fredholm complexes:

$$
\left\{W_{w\left(\delta^{\prime}\right)}^{*}\left(Y(S, 2) ; \Lambda^{*}\right), d^{*}\right\} .
$$

From the above proof, one has the inclusion $H^{2}\left(\delta^{\prime \prime}\right) \subset H^{2}\left(\delta^{\prime}\right)$ for all $0 \leq \delta^{\prime \prime} \leq$ $\delta^{\prime}$. Now let us see $H^{2}\left(\delta^{\prime}\right)=H^{2}(0)$ and $H^{1}(0)=0$. Suppose $H^{1}(0) \neq 0$. Then by invariance of Fredholm indices, one must have $\operatorname{dim} H^{2}(0)>\operatorname{dim} H^{2}\left(\delta^{\prime}\right)$. This contradicts the above. This completes the proof.

\section{C Computation of $H^{2}(\mathrm{AHS})$}

To show that $P_{\tau}: W_{\tau}^{k+1}\left(Y(S, 2) ; \Lambda^{1}\right) \rightarrow W_{\tau}^{k}\left(Y(S, 2) ; \Lambda^{0} \oplus \Lambda_{+}^{2}\right)$ is invertible, one uses an asymptotic method. Roughly speaking one approximates $Y(S, 2)$ by a family of Riemannian manifolds with cylindrical ends. Then the spectrum of $P_{\tau}$ over each cylindrical manifold has a uniform lower bound on 1 forms. From this one gets a lower bound of $\left(d_{+}\right)_{\tau}^{*}$ over $Y(S, 2)$. In the presence of $H^{2}(Y ; \mathbb{R})$, one can get a uniform bound of $\operatorname{dim} H^{2}(\mathrm{AHS})$. The approximation of spaces corresponds to the one of infinite tree by its finite subtrees. This method completely works for higher stages in 5.D. 
Computation of $\operatorname{dim} H^{2}$ (AHS) uses information of $H^{1}$ (AHS) on both approximation spaces and the limit space. The former is obtained by proposition 4.3 and the latter by sublemma 3.1. On the other hand for all cases $H^{0}(\mathrm{AHS})=0$. Then using this and proposition 4.3, one can apply the asymptotic method in this section to verify $H^{1}(\mathrm{AHS})=0$ for the limit space. This will be another method to compute $H^{1}$ (AHS) without using sublemma 3.1.

Let us take $\left(W_{s}=S^{1} \times D^{3}, N_{0}=S^{1} \times D^{2}\right)$ where $N_{0}$ represents a generator of $\pi_{1}\left(W_{s}\right)$. Then by the construction in 2.A, one gets a complete Riemannian manifold $\widetilde{W}_{s}$ with one end $N_{0}([0, \infty))$ along horizontal direction. Let $\left(W_{0}, N_{0}, M_{1}\right)$ be the simplest kinky handle as before. Let us put:

$$
\begin{aligned}
& Y(S)_{0}(n)^{\prime}=\widetilde{W}_{0}^{1}([-S, S]) \cup_{M_{1}^{1}(S) \sim N_{0}^{2}(S)} \widetilde{W}_{0}^{2}([-S, S]) \\
& \quad \ldots \cup_{M_{1}^{n-1}(S) \sim N_{0}^{n}(S)} \widetilde{W}_{0}^{n}([-S, S]), \\
& Y(S)_{0}(n)=Y(S)_{0}(n)^{\prime} \cup_{M_{1}^{n}(S) \sim N_{0}(S)} \widetilde{W}_{s} \backslash N_{0}([S, \infty)), \\
& Y(S)_{0}^{\prime \prime}=\ldots \widetilde{W}_{0}^{-n}([-S, S]) \cup_{M_{1}^{-n}(S) \sim N_{0}^{-n+1}(S)} \widetilde{W}_{0}^{-n+1}([-S, S]) \ldots \\
& \quad \cup_{M_{1}^{n-1}(S) \sim N_{0}^{n}(S)} \widetilde{W}_{0}^{n}([-S, S]) \cup_{M_{1}^{n}(S) \sim N_{0}(S)} \widetilde{W}_{s} \backslash N_{0}([S, \infty)) .
\end{aligned}
$$

Notice $Y(S)_{0}(\infty)^{\prime}=Y(S)_{0}$. Let us put $Y(S, 2)(n)^{\prime}=Y(S, 2)^{\prime} \cup_{M_{2}(S) \sim N_{0}^{0}(S)}$ $Y(S)_{0}(n)^{\prime}$ and:

$$
Y(S, 2)(n)=Y(S, 2)^{\prime} \cup_{M_{2}(S) \sim N_{0}^{0}(S)} Y(S)_{0}(n) .
$$

$Y(S, 2)(n)$ is a complete Riemannian manifold with cylindrical end. Let the weight function be $\tau: Y(S, 2)(n) \rightarrow[0, \infty)$, with weight $\delta$ as before.

Proposition 5.1 One gets isomorphisms:

$$
P_{\tau}: W_{\tau}^{k+1}\left(Y(S, 2)(n) ; \Lambda^{1}\right) \cong W_{\tau}^{k}\left(Y(S, 2)(n) ; \Lambda^{0} \oplus \Lambda_{+}^{2}\right) .
$$

Proof By proposition 4.3, the conclusion is true when one uses a sufficiently small weight constant $\delta^{\prime}$. On the other hand, by the first part of the proof of lemma 5.1, the differential of the AHS complex has closed range for all $\delta^{\prime} \in(0, \delta]$. From this, one gets $H^{0}(\mathrm{AHS})=H^{1}(\mathrm{AHS})=0$ for all $\delta^{\prime}$. To see $H^{2}$ (AHS) $=0$, one can use the same method as proposition 4.2. This completes the proof.

In particular there are constants $C_{n}$ such that for $\Delta=\left(P_{\tau}\right)_{\tau}^{*} \circ P_{\tau}$, one has the bounds $|u| W_{\tau}^{k+2}(Y(S, 2)(n)) \leq C_{n}|\Delta(u)| W_{\tau}^{k}(Y(S, 2)(n))$. Also one has bounds:

$$
\begin{aligned}
|u| W_{\tau}^{k+2}(Y(S, 2)) & \leq C|\Delta(u)| W_{\tau}^{k}(Y(S, 2)), \\
|u| W_{\tau}^{k+2}\left(Y(S)_{0}^{\prime \prime}\right) & \leq C|\Delta(u)| W_{\tau}^{k}\left(Y(S)_{0}^{\prime \prime}\right) .
\end{aligned}
$$


Sublemma 5.2 There is a lower bound $C_{n} \leq C$ for all $n$.

Proof For any small $\epsilon>0$, there exists a large $n$ with the following property: for any $u \in W_{\tau}^{k+2}(Y(S, 2)(n))$ with $|u| W_{\tau}^{k+2}=1$, there is at least one $n^{\prime}$, $0 \leq n^{\prime} \leq n$ with $|u| W_{\tau}^{k+2}\left(\widetilde{W}_{0}^{n^{\prime}}([-S, S])<\epsilon\right.$. Let $\varphi\left(n^{\prime}\right)$ be a cut-off function on $Y(S, 2)(n)$ with $\varphi\left(n^{\prime}\right) \mid\left(\widetilde{W}_{0}^{n^{\prime}}([-S, S])\right)^{c} \equiv 1$ and $Y(S, 2)(n) \backslash \operatorname{Supp} d \varphi\left(n^{\prime}\right)$ has two components. Then one may express $\varphi\left(n^{\prime}\right) u=u_{1}+u_{2}$. One may regard $u_{1} \in W_{\tau}^{k+2}(Y(S, 2)), u_{2} \in W_{\tau}^{k+2}\left(Y(S)_{0}^{\prime \prime}\right)$. In particular one has the estimates:

$$
\begin{aligned}
|u| W_{\tau}^{k+2} & \leq\left|u_{1}\right| W_{\tau}^{k+2}+\left|u_{2}\right| W_{\tau}^{k+2}+\left|\left(1-\varphi\left(n^{\prime}\right)\right) u\right| W_{\tau}^{k+2} \\
& \leq C\left\{\left|\Delta\left(u_{1}\right)\right| W_{\tau}^{k}+\left|\Delta\left(u_{2}\right)\right| W_{\tau}^{k}\right\}+\epsilon \leq C|\Delta(u)| W_{\tau}^{k}+C \epsilon
\end{aligned}
$$

where $C$ is independent of $\epsilon$ and $u$. This gives the result.

Now suppose $H^{2}$ (AHS) is nonzero over $Y(S, 2)$, and take $u \in \operatorname{ker}\left(d_{+}\right)_{\tau}^{*} \cap$ $W_{\tau}^{k+1}\left(Y(S, 2) ; \Lambda_{+}^{2}\right)$ with $|u| W_{\tau}^{k+1}=1$. For each $n$, let us take a cut-off function $\varphi(n)$ on $Y(S, 2)$ with $\varphi(n)\left|Y(S, 2)(n-1)^{\prime} \equiv 1, \varphi(n)\right|\left(Y(S, 2)(n)^{\prime}\right)^{c} \equiv$ 0 . Then one may regard $\varphi(n) u \in W_{\tau}^{k+1}\left(Y(S, 2)(n) ; \Lambda_{+}^{2}\right)$. Thus there exists $v_{n} \in W_{\tau}^{k+2}\left(Y(S, 2)(n) ; \Lambda^{1}\right)$ with $d_{\tau}^{*}\left(v_{n}\right)=0, d_{+}\left(v_{n}\right)=\varphi(n) u$. By the above sublemma, one has the uniform estimates:

$$
\begin{aligned}
& 1-\epsilon \leq|\varphi(n) u| W_{\tau}^{k+1}=\left|d_{+}\left(v_{n}\right)\right| W_{\tau}^{k+1} \\
& \leq C\left|v_{n}\right| W_{\tau}^{k+2} \leq C\left|d_{+}\left(v_{n}\right)\right| W_{\tau}^{k+1} \leq C|\varphi(n) u| W_{\tau}^{k+1} .
\end{aligned}
$$

By the above $(*)$, one has the uniform estimates $C \leq\left|v_{n}\right| W_{\tau}^{k+2} \leq C^{\prime}$. Let us take a sequence $1 \gg \epsilon_{1} \gg \epsilon_{2} \gg \cdots \rightarrow 0$. Then for each $n$, there exists a large $N=N(n)$ and $L(n) \gg 0$ such that at least one $\left|v_{N}\right| W_{\tau}^{k+2}\left(\widetilde{W}_{0}^{n^{\prime}}([-S, S])\right)$ is less than $\epsilon_{n}$ for $N-L(n) \leq n^{\prime} \leq N$. Let us take a subsequence $\left\{v_{N(n)}\right\}_{n}$ and denote it by $\left\{v_{n}\right\}_{n}$. For simplicity of the notation, one may assume $n^{\prime}=n-1$.

Using this, one can verify $\operatorname{ker}\left(d_{+}\right)_{\tau}^{*}=0$ over $Y(S, 2)$. Let us put $\Delta=d d_{\tau}^{*} \oplus$ $\left(d_{+}\right)_{\tau}^{*} d_{+}$on $W_{\tau}^{k+2}\left(Y(S, 2) ; \Lambda^{1}\right)$. From above, one has the estimate:

$$
\begin{aligned}
& \left|d d_{\tau}^{*}\left(\varphi(n-1) v_{n}\right)\right| W_{\tau}^{k} \\
& \quad \leq C\left\{\left|d v_{n}\right| W_{\tau}^{k}\left(\widetilde{W}_{0}^{n-1}([-S, S])\right)+\left|v_{n}\right| W_{\tau}^{k}\left(\widetilde{W}_{0}^{n-1}([-S, S])\right)\right\} \leq C \epsilon_{n}
\end{aligned}
$$


where $C$ is independent of $n$. Then one has the estimates:

$$
\begin{aligned}
& \left|\Delta\left(\varphi(n-1) v_{n}\right)\right| W_{\tau}^{k} \leq C\left[\left|\left(d_{+}\right)_{\tau}^{*}(\varphi(n-1) \varphi(n) u)\right| W_{\tau}^{k}\right. \\
& \left.\quad+\left|\left(d_{+}\right)_{\tau}^{*}\left[d_{+}, \varphi(n-1)\right] v_{n}\right| W_{\tau}^{k}(\operatorname{Supp} d \varphi(n-1))\right]+C \epsilon_{n} \\
& \leq C \\
& \quad\left[\left|\varphi(n-1) \varphi(n)\left(d_{+}\right)_{\tau}^{*}(u)\right| W_{\tau}^{k}\right. \\
& \quad+\left|\left[\left(d_{+}\right)_{\tau}^{*}, \varphi(n-1) \varphi(n)\right] u\right| W_{\tau}^{k}\left(\cup_{j=n-1, n} \widetilde{W}_{0}^{j}([-S, S])\right) \\
& \quad+\left|\left(d_{+}\right)_{\tau}^{*}\left[d_{+}, \varphi(n-1)\right] v_{n}\right| W_{\tau}^{k}\left(\widetilde{W}_{0}^{n-1}([-S, S])\right)+C \epsilon_{n} \\
& \quad \leq\left|\left[\left(d_{+}\right)_{\tau}^{*}, \varphi(n-1) \varphi(n)\right] u\right| W_{\tau}^{k}+\left|\left(d_{+}\right)_{\tau}^{*}\left[d_{+}, \varphi(n-1)\right] v_{n}\right| W_{\tau}^{k}+C \epsilon_{n} .
\end{aligned}
$$

One may assume that the last term is arbitrarily small. This shows that there is a sequence $\left\{w_{n} \in W_{\tau}^{k+2}\left(Y(S, 2) ; \Lambda^{1}\right)\right\}$ with $C \leq\left|w_{n}\right| W_{\tau}^{k+2} \leq C^{\prime}$ and $\left|P\left(w_{n}\right)\right| W_{\tau}^{k+1}$ converges to zero. This is a contradiction. This shows $\operatorname{ker}\left(d_{+}\right)_{\tau}^{*}=0$ over $Y(S, 2)$.

Let $\left(W_{t}, N_{0}\right)=\left(D^{4}, S^{1} \times D^{2}\right)$ be the standard disk. Let $Y=\widetilde{W}_{t} \backslash N_{0}([S, \infty)) \cup$ $\widetilde{Y}(S)_{0} \backslash N_{0}([S, \infty))$. In this case, one has $H^{0}$ (AHS) $=H^{1}$ (AHS) $=0$ (notice $Y$ is simply connected). But $H^{2}(Y ; \mathbb{R})=\mathbb{R}$. In this case, one has the following:

Corollary 5.1 Suppose $Y$ has nonzero $H^{2}(Y ; \mathbb{R})$. Then one has a bound $\operatorname{dim} H^{2}(\mathrm{AHS}) \leq 2 \operatorname{dim} H^{2}(Y ; \mathbb{R})$.

Proof Suppose $\operatorname{dim} H^{2}(\mathrm{AHS}) \geq 2 \operatorname{dim} H^{2}(Y ; \mathbb{R})+1=b_{2}+1$. Let us take $L_{\tau}^{2}$ orthogonal vectors $u_{1}, \ldots, u_{b_{2}+1}, \cdots \in L_{\tau}^{2}\left(Y: \Lambda_{+}^{2}\right) \cap \operatorname{ker}\left(d_{+}\right)_{\tau}^{*}$ with $\left|u_{i}\right| L_{\tau}^{2}=1$.

One can make a family of cylindrical manifolds $Y(n)$ by the above method. By a straightforward calculation, one has an upper bound of $\operatorname{dim} H^{2}(Y(n) ; \mathbb{R})$ by $b_{2}$. By remark 4.2, it follows that $\operatorname{dim} H^{2}$ (AHS) has also an upper bound over $Y(n)$. Now let $\varphi(n)$ be as above. Then for any small $\epsilon>0$, there exists a large $n_{0}$ such that for all $n \geq n_{0}$, one has:

$$
\begin{aligned}
& \left|\left(d_{+}\right)_{\tau}^{*}\left(\varphi(n) u_{i}\right)\right| L_{\tau}^{2} \leq \epsilon, \quad\left|\varphi(n) u_{i}\right| L_{\tau}^{2} \geq 1-\epsilon \\
& \left\langle\varphi(n) u_{i}, \varphi(n) u_{j}\right\rangle \mid L_{\tau}^{2} \leq \epsilon, \quad i=1, \ldots, b_{2}+1
\end{aligned}
$$

Let $v_{1}, \ldots, v_{l}$ be an orthonormal basis of $H^{2}(\mathrm{AHS})$ over $Y(n), l \leq b_{2}$. Then one may express $\varphi(n) u_{i}=\Sigma_{j} a_{j}^{i} v_{j}+d_{+}\left(\alpha_{i}\right), a_{j}^{i} \in \mathbb{R}$. Let $\bar{a}_{i}=\left(a_{1}^{i}, \ldots, a_{l}^{i}\right) \in \mathbb{R}^{l}$. Then the set of vectors $\bar{a}_{1}, \ldots, \bar{a}_{b_{2}+1}$ would satisfy $\left|\bar{a}_{i}\right| \geq 1-\epsilon$ and $\left\langle\bar{a}_{i}, \bar{a}_{i^{\prime}}\right\rangle \leq \epsilon$ with respect to the standard norm in $\mathbb{R}^{l}$. Since $l<b_{2}+1$, it is impossible to find such set. This completes the proof. 


\section{D Fourier-Laplace transforms on higher stages}

Let us put:

$$
\begin{aligned}
& \widetilde{W}_{2}([-S, S])=\widetilde{W}_{2} \backslash N_{0}([S, \infty)) \cup_{j=1,2} M_{j}([S, \infty)), \\
& Y(S, 2)_{0}=\widetilde{W}_{2} \cup_{M_{2}(S) \sim N_{0}(S)} \widetilde{Y}(S)_{0} \backslash N_{0}([S, \infty)), \\
& \widetilde{Y}(S, 2)=\ldots Y(S, 2)_{0}^{-n} \cup_{M_{1}^{-n}(S) \sim N_{0}^{-n+1}(S)} Y(S, 2)_{0}^{-n+1} \ldots \\
& \widetilde{Y}(S, 2)_{0}=Y(S, 2)_{0}^{1} \cup_{M_{1}^{1}(S) \sim N_{0}^{2}(S)} Y(S, 2)_{0}^{2} \cup_{M_{1}^{2}(S) \sim N_{0}^{3}(S)} Y(S, 2)_{0}^{3} \ldots
\end{aligned}
$$

where $\left(Y(S, 2)_{0}^{n}, M_{j}^{n}(S), N_{0}^{n}(S)\right)$ is the same triple $\left(Y(S, 2)_{0}, M_{j}(S), N_{0}(S)\right)$ as before. Notice that $\tilde{Y}(S, 2)_{0}$ is diffeomorphic to $C H\left(T_{2}^{0}\right)$, where $T_{2}^{0}=$ $\mathbb{R}_{+} \cup_{n \in \mathbb{N}} \mathbb{R}_{+}$. Now by the Fourier-Laplace transform, one gets the following:

Corollary $5.2 P_{\tau}: W_{\tau}^{k+1}\left(\widetilde{Y}(S, 2) ; \Lambda^{1}\right) \rightarrow W_{\tau}^{k}\left(\widetilde{Y}(S, 2) ; \Lambda^{0} \oplus \Lambda_{+}^{2}\right)$ is invertible.

Now one defines: $T_{3}=\mathbb{R} \cup_{n \in \mathbb{Z}} T_{2}^{0}$ and $T_{3}^{0}=\mathbb{R}_{+} \cup_{n \in \mathbb{N}} T_{2}^{0}$. Similarly $T_{4}=$ $\mathbb{R} \cup_{n \in \mathbb{Z}} T_{3}^{0}$. One inductively defines $T_{j}, j=1,2, \ldots$ as:

$$
T_{j+1}=\mathbb{R} \cup_{n \in \mathbb{Z}} T_{j}^{0}, \quad T_{j+1}^{0}=\mathbb{R}_{+} \cup_{n \in \mathbb{N}} T_{j}^{0}
$$

where one puts $T_{1}=\mathbb{R}, T_{1}^{0}=\mathbb{R}_{+}$.

One can construct the corresponding spaces. Let $\left(W_{2}, N_{0}, M_{1}, M_{2}\right)$ be a kinky handle with two kinks. One has already defined $Y(S), Y(S, 2)$. Let us define inductively $Y(S, j)$ as follows:

$$
\begin{aligned}
& Y(S, j)_{0}=\widetilde{W}_{2}([-S, S]) \cup_{M_{2}(S) \sim N_{0}(S)} \tilde{Y}(S, j-1)_{0}, \\
& Y(S, j)=Y(S, j)_{0} / N_{0}(S) \sim M_{1}(S), \\
& \widetilde{Y}(S, j)=\ldots Y(S, j)_{0}^{-n} \cup_{M_{1}^{-n}(S) \sim N_{0}^{-n+1}(S)} Y(S, j)_{0}^{-n+1} \ldots \\
& \widetilde{Y}(S, j)_{0}=Y(S, j)^{1} \cup_{M_{1}^{1}(S) \sim N_{0}^{2}(S)} Y(S, j)^{2} \cup_{M_{1}^{2}(S) \sim N_{0}^{3}(S)} Y(S, j)^{3} \ldots
\end{aligned}
$$

One may express $C H\left(T_{j}^{0}\right)=\tilde{Y}(S, j)_{0}$. The previous method works for all $Y(S, j)$ iteratively.

Proposition 5.2 $P_{\tau}$ over $Y(S, j)$ are all Fredholm with $H^{*}(\mathrm{AHS})=0$ for $*=0,1,2$ and $j=1,2, \ldots$.

In the notation in 2.B, one expresses $T_{j}^{0}=\left(T_{2,2, \ldots, 2,1}\right)_{0}((2, \ldots, 2) j-1$ times $)$. Let $n_{1}, \ldots, n_{k} \in\{1,2, \ldots\}$ be a set of positive integers. Then using kinky handles with $n_{j}$ kinks, one has a natural extension, and gets $\left(T_{n_{1}, \ldots, n_{k}, 1}\right)_{0}$ which is a signed infinite tree. For Riemannian metrics on $C H\left(\left(T_{n_{1}, \ldots, n_{k}, 1}\right)_{0}\right)$, see 2.B. 
Let $\bar{n}=\left\{n_{1}, \ldots, n_{l-1}, 1\right\}$ be a set of positive integers, and denote the corresponding homogeneous tree of bounded type by $\left(T_{\bar{n}}\right)_{0}$. By the previous method, one gets a complete Riemannian metric and a weight function on every $\mathrm{CH}\left(\left(T_{\bar{n}}\right)_{0}\right)$. Recall that one has constructed complete Riemannian metrics and weight functions on $Y(S, \bar{n})$.

Proposition $5.3 P_{\tau}: W_{\tau}^{k+1}\left(Y(S, \bar{n}) ; \Lambda^{1}\right) \rightarrow W_{\tau}^{k}\left(Y(S, \bar{n}) ; \Lambda^{0} \oplus \Lambda_{+}^{2}\right)$ gives an isomorphism for any $\bar{n}=\left\{n_{1}, \ldots, n_{l-1}, 1\right\}$.

Proof One has shown the result for $l=2$. Suppose the result is true for $l \leq l_{0}$. Let us put $\bar{n}=\left\{n_{1}, \ldots, n_{l_{0}+1}, 1\right\}$. Then by Fourier-Laplace transform and excision method used before, one knows $P_{\tau}: W_{\tau}^{k+1}\left(Y(S, \bar{n}) ; \Lambda^{1}\right) \rightarrow$ $W_{\tau}^{k}\left(Y(S, \bar{n}) ; \Lambda^{0} \oplus \Lambda_{+}^{2}\right)$ gives a closed operator with $H^{*}(\mathrm{AHS})=0$ for $*=0,1$. One may follow the same process to see $H^{2}$ (AHS) $=0$ as 5.C. Thus one has shown the result for $l_{0}+1$. This completes the induction step.

In practical applications, one considers open four-manifolds composed of one 0 -handle attached with Casson handles. Recall that $k\left(S^{2} \times S^{2}\right) \backslash p t$ is homotopyequivalent to some wedges of $S^{2}$. In particular $H_{\mathrm{cp}}^{1}(M ; \mathbb{R})=H^{3}(M ; \mathbb{R})=0$. Recall also that it has a link picture by $k$ disjoint union of Hopf links with 0-framings. Let $\left(W_{t}, M_{1}, \ldots, M_{2 k}\right)=\left(D^{4}, S^{1} \times D^{2}, \ldots, S^{1} \times D^{2}\right)$ express the link diagram of $k\left(S^{2} \times S^{2}\right) \backslash p t$.

Let $\left(T_{1}\right)_{0}, \ldots,\left(T_{2 k}\right)_{0}$ be signed homogeneous trees of bounded type. Let us consider an open four-manifold $S$ obtained by attaching $C H\left(\left(T_{i}\right)_{0}\right)$ along $\left(D^{4}, S^{1} \times D^{3}, \ldots, S^{1} \times D^{3}\right)$. Then by the previous procedure, one can equip a complete Riemannian metric on $S$ :

$$
S \equiv \widetilde{W}_{t} \natural \cup_{l} C H\left(\left(T_{l}\right)_{0}\right) .
$$

As before one can also equip a weight function $\tau$, and the AHS complex over $S$.

Corollary 5.3 The differential of AHS complex has closed range over $S$ with $H^{0}(\mathrm{AHS})=H^{1}(\mathrm{AHS})=0$ and $\operatorname{dim} H^{2}(\mathrm{AHS}) \leq 2 \operatorname{dim} H^{2}(S ; \mathbb{R})$.

By the work of Freedman [15], the end of $S$ admits a topological color, $\cong$ $S^{3} \times[0, \infty)$. In fact $S$ is homeomorphic to $k\left(S^{2} \times S^{2}\right) \backslash p t$. Now we have completed the verification that any open four-manifold with a tree-like end of bounded type can admit an admissible pair $(g, \tau)$ on it. 


\section{References}

[1] M Atiyah, N Hitchin, I Singer, Self-duality in four dimensional Riemannian geometry, Proc. R. Soc. London 362 (1978) $425-461$

[2] M Atiyah, V Patodi, I Singer, Spectral asymmetry in Riemannian geometry, I, II and III, Math. Proc. Cambridge Philos. Soc. 77 (1975) 43 - 69; 78 (1975) 405-432; 79 (1976) 71-99

[3] Ž Bižaca, A re-imbedding algorithm for Casson handles, Trans. A.M.S. 345 (1994) 435-510

[4] Ž Bižaca, An explicit family of exotic Casson handles, Proc. of the A.M.S. 123 (1995) 1297-1302

[5] Ž Bižaca, R Gompf, Elliptic surfaces and some simple exotic $\mathbb{R}^{4}$ 's, Journal of Differential Geometry 43 (1996) 458-504

[6] A Casson, Three lectures on new infinite constructions in 4-dimensional manifolds,

[7] A Connes, Non-commutative geometry, Academic press (1994)

[8] S Demichelis, M Freedman, Uncountably many exotic $\mathbb{R}^{4}$ 's in standard 4space, Journal of Differential Geometry 35 (1992) 219-254

[9] S Donaldson, Polynomial invariants for smooth 4-manifolds, Topology 29 (1990) 257-315

[10] S Donaldson, An application of gauge theory to four-dimensional topology, Journal of Differential Geometry 18 (1983) 279-315

[11] S Donaldson, P Kronheimer, The geometry of four-manifolds, Oxford University Press (1990)

[12] Y Eliashberg, Topological characterization of Stein manifolds of dimension $>2$, Internat. J. Math. 98 (1990) 29-46

[13] A Floer, An instanton invariant for 3 manifolds, Comm.Math.Phy. 118 (1988) 215-240

[14] S Freed, K Uhlenbeck Instantons and four-manifolds, Springer, 2nd edition (1991)

[15] M Freedman, The topology of four-dimensional manifolds, Journal of Differential Geometry 17 (1982) 357-454

[16] M Freedman, F Quinn, Topology of 4-manifolds, Princeton Univ. Press (1990)

[17] M Freedman, L Taylor, A universal smoothing of four-space, Journal of Differential Geometry 24 (1986) 69-78

[18] R Gompf, Handlebody construction of Stein surfaces, Ann. Math. 148 (1998) 619-693 
[19] R Gompf, A Stipsicz, 4-manifolds and Kirby calculus, Volume 20 of Graduate Studies in Mathematics, AMS (1999)

[20] M Gromov, H Lawson, Positive scalar curvature on complete Riemannian manifolds, I.H.E.S Publ. Math. 58 (1983) 295-408

[21] T Kato, Spectral analysis on tree like spaces from gauge theoretic aspects, to appear in the Proceedings of Discrete Geometric Analysis, Contemporary Math., AMS

[22] R Kirby, The topology of 4-manifolds, Volume 1374 of Lecture Notes in Mathematics, Springer-Verlag

[23] V Kondrat'ev Boundary value problems for elliptic equations in domains with conical or angular points, Trans. Moscow Math. Soc. 16 (1967)

[24] R Lockhart, R McOwen, Elliptic differential operators on non compact manifolds, Ann. Sci. Ec. Norm. Sup. Pisa 12 (1985) 409-446

[25] J Milnor On simply connected 4-manifolds, Symp. Int. Top. Alg. Mexico (1958) $122-128$

[26] F Quinn, Ends of maps in dimension 4 and 5 Journal of Differential Geometry 17 (1982) 503-521

[27] C Taubes, Gauge theory on asymptotically periodic 4 manifolds, Journal of Differential Geometry 25 (1987) 363-430

[28] C Taubes, Casson's invariant and gauge theory, Journal of Differential Geometry 31 (1990) 547-599

[29] L Taylor An invariant of smooth 4-manifolds, Geometry and Topology 1 (1997) 71-89

[30] K Uhlenbeck Connections with $L^{p}$ bounds on curvature, Comm. Math. Phys. 83 (1982) 31-42

[31] K Uhlenbeck Removable singularities in Yang-Mills fields, C.M.P. 83 (1982) 11-29

[32] F Warner Foundations of differentiable manifolds and Lie groups, Graduate Texts in Mathematics, Springer-Verlag (1971). 